\title{
Output Feedback Adaptive Stabilization of Uncertain Nonholonomic Systems
}

\author{
Yuanyuan Wu, ${ }^{1,2}$ Zicheng Wang, ${ }^{1}$ Yuqiang $\mathrm{Wu},{ }^{3}$ and Qingbo $\mathrm{Li}^{4}$ \\ ${ }^{1}$ College of Electric and Information Engineering, Zhengzhou University of Light Industry, Zhengzhou, Henan 450002, China \\ ${ }^{2}$ Department of Mathematics, Southeast University, Nanjing, Jiangsu 210096, China \\ ${ }^{3}$ Research Institute of Automation, Qufu Normal University, Qufu, Shandong 273165, China \\ ${ }^{4}$ College of Mathematics and Information Science, Zhengzhou University of Light Industry, Zhengzhou, Henan 450002, China
}

Correspondence should be addressed to Qingbo Li; qbliyy@163.com

Received 23 January 2014; Accepted 15 April 2014; Published 12 May 2014

Academic Editor: Hao Shen

Copyright (C) 2014 Yuanyuan Wu et al. This is an open access article distributed under the Creative Commons Attribution License, which permits unrestricted use, distribution, and reproduction in any medium, provided the original work is properly cited.

\begin{abstract}
This paper investigates the problem of output feedback adaptive stabilization control design for a class of nonholonomic chained systems with uncertainties, involving virtual control coefficients, unknown nonlinear parameters, and unknown time delays. The objective is to design a robust nonlinear output-feedback switching controller, which can guarantee the stabilization of the closed loop systems. An observer and an estimator are employed for states and parameters estimates, respectively. A constructive controller design procedure is proposed by applying input-state scaling transformation, parameter separation technique, and backstepping recursive approach. Simulation results are provided to show the effectiveness of the proposed method.
\end{abstract}

\section{Introduction}

The control and feedback stabilization problems of nonholonomic systems have been widely studied by many researchers. It is well known that control of nonholonomic systems is extremely challenging, largely due to the impossibility of asymptotically stabilizing nonholonomic systems via smooth time-invariant state feedback, a well-recognized fact pointed out in $[1,2]$. In order to overcome this obstruction, a number of approaches have been proposed for the problem, which mainly include discontinuous feedback, time-varying feedback, and hybrid stabilization. The discontinuous feedback stabilization was first proposed by [3], and then further discussion was made in [4-7]; especially an elegant discontinuous coordinate transformation approach is proposed in [5] for the stabilization problem of nonholonomic systems. Meanwhile, the smooth time-varying feedback control strategies also have drawn much attention [8-11].

As pointed out in [9], many nonlinear mechanical systems with nonholonomic constraints can be transformed, either locally or globally, to the nonholonomic systems in the so-called chained form. So far, there have been a number of controller design approaches [8-25] for such chained nonholonomic systems. Recently, adaptive control strategies have been proposed to stabilize the nonholonomic systems. For instance, the problem of adaptive state-feedback control is studied in [15-19], while output feedback controller design in [20-24]. Considering the actual modeling perspective, time delay should be taken into account. The problem of state feedback stabilization is studied for the delayed nonholonomic systems in $[25,26]$. However, the virtual control coefficients and unknown parameter vector are not considered in its system models. Here, an iterative controller design method will be proposed for the output feedback adaptive stabilization of the concerned delayed nonholomic systems.

In this paper, we study a class of chained nonholonomic systems with strong nonlinear drifts, and the problem of adaptive output-feedback stabilization for the concerned nonholonomic systems is investigated. The constructive design method proposed in this note is based on a combined application of the input scaling technique, the backstepping 
recursive approach, and the novel Lyapunov-Krasovskii functionals. The switching control strategy for the first subsystem is employed to achieve the asymptotic stabilization.

The rest of this paper is organized as follows. In Section 2, the problem formulation and some preliminary knowledge are given. Section 3 presents the controller design procedure and stability analysis. Section 4 gives the switching control strategy. In Section 5, numerical simulations testify to the effectiveness of the proposed method, and Section 6 summarizes the paper.

\section{Problem Formulation and Preliminaries}

In this paper, we deal with a class of nonholonomic systems described by

$$
\begin{aligned}
\dot{x}_{0}(t)= & d_{0} u_{0}(t)+\phi_{0}\left(t, x_{0}(t)\right), \\
\dot{x}_{1}(t)= & d_{1} u_{0}(t) x_{2}(t)+\varphi_{1}\left(u_{0}(t), y(t), y\left(t-\tau_{1}\right)\right) \\
& +\phi_{1}\left(t, u_{0}(t), x_{0}(t), x(t), \theta\right), \\
\vdots & \\
\dot{x}_{n-1}(t)= & d_{n-1} u_{0}(t) x_{n}(t)+\varphi_{n-1}\left(u_{0}(t), y(t), y\left(t-\tau_{n-1}\right)\right) \\
& +\phi_{n-1}\left(t, u_{0}(t), x_{0}(t), x(t), \theta\right), \\
\dot{x}_{n}(t)= & d_{n} u_{1}(t)+\varphi_{n}\left(u_{0}(t), y(t), y\left(t-\tau_{n}\right)\right) \\
& +\phi_{n}\left(t, u_{0}(t), x_{0}(t), x(t), \theta\right), \\
y(t)= & {\left[x_{0}(t), x_{1}(t)\right]^{T}, }
\end{aligned}
$$

where $\left[x_{0}(t), x(t)\right]^{T}=\left[x_{0}(t), x_{1}(t), \ldots, x_{n}(t)\right]^{T} \in R^{n+1}$, $u(t)=\left[u_{0}(t), u_{1}(t)\right]^{T} \in R^{2}$, and $y(t) \in R^{2}$ are system states, control input, and measurable output, respectively; $\theta \in R^{m}$ is an unknown parameter vector; $\phi_{0}$ (known) and $\phi_{i}(1 \leq i \leq n)$ (unknown) denote the possible modeling error and neglected dynamics; $\varphi_{i}(1 \leq i \leq n)$ are known modeled dynamics, which contain output delays; $\tau_{i}(1 \leq i \leq n)$ are unknown constants, and $d_{i}(0 \leq i \leq n)$ referred to the respective virtual control coefficients.

In this paper, we make the following assumptions on the virtual control directions $d_{i}$ and nonlinear functions $\varphi_{i}, \phi_{i}$ in system (1).

Assumption 1. $d_{0}$ is a known constant and the sign of $\bar{d}_{n}$ is known, where $\bar{d}_{n}=d_{1} d_{2} \cdots d_{n}$.

Assumption 2. There exist known smooth nonnegative functions $\bar{\phi}_{o}$ and $\bar{\phi}_{i}(1 \leq i \leq n)$ such that

$$
\begin{gathered}
\phi_{0}\left(t, x_{0}(t)\right)=x_{0} \bar{\phi}_{0}\left(x_{0}(t)\right), \\
\left|\phi_{i}\left(t, u_{0}(t), x_{0}(t), x(t), \theta\right)\right| \\
\leq\left|x_{1}\right| \bar{\phi}_{i}\left(u_{0}(t), x_{0}(t), x_{1}(t), \theta\right) .
\end{gathered}
$$

for all $\left(t, u_{0}(t), x_{0}(t), x(t), \theta\right) \in R_{+} \times R \times R \times R^{n} \times R^{m}$.
Assumption 3. For every $1 \leq i \leq n$, the nonlinear function $\varphi_{i}$ satisfies inequality

$$
\begin{aligned}
\left|\varphi_{i}\left(u_{0}(t), y(t), y\left(t-\tau_{i}\right)\right)\right| \leq & \left|x_{1}(t)\right| \psi_{i}\left(u_{0}(t), y(t)\right) \\
& +\left|x_{1}(t) x_{1}\left(t-\tau_{i}\right)\right| \\
& \times \bar{\varphi}_{i}\left(u_{0}(t), y(t), y\left(t-\tau_{i}\right)\right),
\end{aligned}
$$

in which $\bar{\varphi}_{i}$ and $\psi_{i}$ are known smooth nonnegative nonlinear functions.

Remark 4. Compared with some existing literatures in recent years, the structure of our concerned system (1) is more general. For instance, in [15], it is assumed that not only the virtual control directions $d_{i}=1$ and the dynamics $\phi_{i}$ satisfy $\phi_{i}=\widetilde{\phi}_{i}^{T} \theta$, but also the modeled dynamics $\varphi_{i}$ do not exist. In [22], the virtual control coefficients and time delays have not been considered, and the expression $\phi_{i}=\widetilde{\phi}_{i}^{T} \theta$ is also required. While $d_{i}=1$ and $\varphi_{i}$ and unknown parameters $\theta$ are not existent, system (1) degenerates to the one studied in [21]. When $\varphi_{i}=0$, together with $\phi_{i}=\widetilde{\phi}_{i}^{T} \theta$, system (1) becomes the considered system in [23].

Remark 5. Note that here we only use the sign of $\bar{d}_{n}=$ $d_{1} d_{2} \cdots d_{n}$ without any knowledge of individual virtual control direction $d_{i}(1 \leq i \leq n)$. Moreover, Assumptions 2 and 3 are imposed on the nonlinear functions $\phi_{i}$ and $\varphi_{i}$, respectively. In fact, if the modeled dynamics $\varphi_{i}$ do not involve time delays, inequality (3) is reduced into

$$
\left|\varphi_{i}\left(u_{0}(t), y(t)\right)\right| \leq\left|x_{1}(t)\right| \psi_{i}\left(u_{0}(t), y(t)\right) .
$$

It can be seen that the above inequality condition is used in some existing literatures, such as $[20,21]$, and so on.

Our object of this paper is to design adaptive output feedback control laws under Assumptions 1-3, such that the system states $\left(x_{0}(t), x(t)\right)$ converge to zero, while other signals of the closed-loop system are bounded. The designed control laws can be expressed in the following form:

$$
\begin{gathered}
u_{0}=\mu_{0}(y(t)), \quad u_{1}=\mu_{1}(y(t), v(t)), \\
\dot{v}(t)=\varrho(y(t), \nu(t)) .
\end{gathered}
$$

Next, we list some lemmas which will be applied in the coming controller design.

Lemma 6 (see [27]). For any real-valued continuous function $f(x, y)$, where $x \in R^{n}, y \in R^{m}$, there are smooth functions $a(x) \geq 0, b(y) \geq 0, c(x) \geq 1, d(y) \geq 1$ such that

$$
|f(x, y)| \leq a(x)+b(y), \quad|f(x, y)| \leq c(x) d(y) .
$$

Lemma 7 (see [19]). For any continuous function $\mu_{0}(t)$ there exist two strictly positive real numerates $p_{\min }$ and $p_{\max }$ such that the unique solution $P(t)$ of the following matrix differential equation: 


$$
\begin{gathered}
\dot{P}=P\left(A-\mu_{0}(t) L\right)^{T}+\left(A-\mu_{0}(t) L\right) P-P C^{T} C P+I, \\
P(0)=P_{0}>0,
\end{gathered}
$$

satisfies $p_{\min } I \leq P(t) \leq p_{\max } I, t \geq 0$.

By Lemma 6 and Assumption 1, we know that there exist smooth functions $\omega_{i} \geq 1$, and $\zeta_{i} \geq 1$ such that

$$
\begin{aligned}
& \left|\phi_{i}\left(t, u_{0}(t), x_{0}(t), x(t), \theta\right)\right| \\
& \quad \leq\left|x_{1}\right| \omega_{i}\left(u_{0}(t), x_{0}(t), x_{1}(t)\right) \zeta_{i}(\theta) .
\end{aligned}
$$

Furthermore, we denote $\vartheta=\sum_{i=1}^{n} \zeta_{i}(\theta)$; then it yields

$$
\begin{aligned}
& \left|\phi_{i}\left(t, u_{0}(t), x_{0}(t), x(t), \theta\right)\right| \\
& \quad \leq\left|x_{1}\right| \omega_{i}\left(u_{0}(t), x_{0}(t), x_{1}(t)\right) \vartheta .
\end{aligned}
$$

\section{Output Feedback Adaptive Stabilization Control Design}

In this paper, we design control laws $u_{0}(t)$ and $u_{1}(t)$ separately to globally asymptotically stabilize the system (1). According to the structure of system (1), we can see that when $x_{0}(t)$ converges to zero, $x_{i}(t)(1 \leq i \leq n)$ will be uncontrollable. A widely used method to design control law $u_{1}(t)$ is to introduce a discontinuous input scaling transformation (12). On the other hand, the control directions $d_{i}$ are unknown; then we should employ another coordinate transformation to overcome the obstacle.

3.1. State Coordinate Transformation. Firstly, we design the coordinate transformation as follows:

$$
\bar{x}_{i}(t)=\bar{d}_{i-1} x_{i}(t), \quad 1 \leq i \leq n,
$$

where $\bar{d}_{0}=1$ and $\bar{d}_{i-1}=d_{1} d_{2} \cdots d_{i-1}(1 \leq i \leq n+1)$. Then, the system (1) can be transformed into

$$
\begin{aligned}
\dot{x}_{0}(t)= & d_{0} u_{0}(t)+\phi_{0}\left(t, x_{0}(t)\right), \\
\dot{\bar{x}}_{1}(t)= & u_{0}(t) \bar{x}_{2}(t)+\varphi_{1}\left(u_{0}(t), y(t), y\left(t-\tau_{1}\right)\right) \\
& +\phi_{1}\left(t, u_{0}(t), x_{0}(t), x(t), \theta\right), \\
& \vdots \\
\dot{\bar{x}}_{n}(t)= & \bar{d}_{n} u_{1}(t)+\bar{d}_{n-1} \varphi_{n}\left(u_{0}(t), y(t), y\left(t-\tau_{n}\right)\right) \\
& +\bar{d}_{n-1} \phi_{n}\left(t, u_{0}(t), x_{0}(t), x(t), \theta\right) .
\end{aligned}
$$

Next, the following input-state scaling discontinuous transformation is introduced:

$$
z_{i}(t)=\frac{\bar{x}_{i}(t)}{u_{0}^{n-i}(t)}, \quad 1 \leq i \leq n .
$$

Under the new $z(t)$-coordinates, the $\bar{x}(t)$-subsystem (10) is changed into

$$
\begin{aligned}
\dot{z}_{1}(t)= & z_{2}(t)-(n-1) \frac{\dot{u}_{0}(t)}{u_{0}(t)} z_{1}(t) \\
& +\frac{1}{u_{0}^{n-1}(t)}\left(\varphi_{1}+\phi_{1}\right), \\
\dot{z}_{i}(t)= & z_{i+1}(t)-(n-i) \frac{\dot{u}_{0}(t)}{u_{0}(t)} z_{i}(t) \\
& +\frac{1}{u_{0}^{n-i}(t)}\left(\bar{d}_{i-1} \varphi_{i}+\bar{d}_{i-1} \phi_{i}\right), \\
\dot{z}_{n}(t)= & \bar{d}_{n} u_{1}(t)+\bar{d}_{n-1} \varphi_{n}+\bar{d}_{n-1} \phi_{n} .
\end{aligned}
$$

Next, we can design the control laws $u_{0}(t)$ and $u_{1}(t)$ to asymptotically stabilize the states $x_{0}(t)$ and $z(t)$, respectively. Rewrite system (13) in the compact form

$$
\dot{z}(t)=\left(A-L \frac{\dot{u}_{0}(t)}{u_{0}(t)}\right) z(t)+B u_{1}(t)+\Psi+\Phi,
$$

where

$$
\begin{aligned}
& A=\left[\begin{array}{cc}
0 & I_{n-1} \\
0 & 0
\end{array}\right], \quad L=\left[\begin{array}{cccc}
n-1 & \cdots & 0 & 0 \\
\vdots & \ddots & \vdots & \vdots \\
0 & \cdots & 1 & 0 \\
0 & \cdots & 0 & 0
\end{array}\right], \\
& B=\left[\begin{array}{c}
0 \\
\vdots \\
0 \\
\bar{d}_{n}
\end{array}\right], \quad \Psi=\left[\begin{array}{c}
\Psi_{1} \\
\Psi_{2} \\
\vdots \\
\Psi_{n}
\end{array}\right], \quad \Phi=\left[\begin{array}{c}
\Phi_{1} \\
\Phi_{2} \\
\vdots \\
\Phi_{n}
\end{array}\right]
\end{aligned}
$$

with

$$
\begin{aligned}
& \Psi_{i}=\bar{d}_{i-1} \frac{\varphi_{i}\left(u_{0}(t), y(t), y\left(t-\tau_{i}\right)\right)}{u_{0}^{n-i}(t)}, \\
& \Phi_{i}=\bar{d}_{i-1} \frac{\phi_{i}^{d}\left(t, u_{0}(t), x_{0}(t), x(t), \theta\right)}{u_{0}^{n-i}(t)} .
\end{aligned}
$$

In order to obtain the estimation for the nonlinear functions $\Psi_{i}$ and $\Phi_{i}$, the following lemmas are given.

Lemma 8. For every $1 \leq i \leq n$, there exists smooth nonnegative function $\widetilde{\omega}_{i}\left(u_{0}(t), x_{0}(t), z_{1}(t)\right)$ such that

$$
\left|\Phi_{i}\right| \leq\left|\bar{d}_{i-1}\right| \cdot\left|z_{1}(t)\right| \widetilde{\omega}_{i}\left(u_{0}(t), x_{0}(t), z_{1}(t)\right) \vartheta .
$$

Lemma 9. For every $1 \leq i \leq n$, there exist smooth nonnegative functions $\tilde{\psi}_{i}, \widetilde{\varphi}_{i}, f_{i 1}, f_{i 2}$ such that

$$
\begin{aligned}
\left|\Psi_{i}\right| \leq & \left|\bar{d}_{i-1}\right| \cdot\left|z_{1}(t) z_{1}\left(t-\tau_{i}\right)\right| \\
& \times \widetilde{\varphi}_{i}\left(u_{0}(t), u_{0}\left(t-\tau_{i}\right), y(t), y\left(t-\tau_{i}\right)\right) \\
& +\left|\bar{d}_{i-1}\right| \cdot\left|z_{1}(t)\right| \widetilde{\psi}_{i}\left(u_{0}(t), y(t)\right) \\
\leq & \left|\bar{d}_{i-1}\right| \cdot\left|z_{1}(t) z_{1}\left(t-\tau_{i}\right)\right| f_{i 1}\left(u_{0}(t), y(t)\right)
\end{aligned}
$$




$$
\begin{aligned}
& \times f_{i 2}\left(u_{0}\left(t-\tau_{i}\right), y\left(t-\tau_{i}\right)\right) \\
& +\left|\bar{d}_{i-1}\right| \cdot\left|z_{1}(t)\right| \tilde{\psi}_{i}\left(u_{0}(t), y(t)\right) .
\end{aligned}
$$

Remark 10. By lemmas and assumptions before, Lemmas 8 and 9 can be derived easily, and then the proof is omitted.

3.2. Observer Design. Define the following filter/estimator:

$$
\begin{gathered}
\dot{\xi}_{0}(t)=\left(A_{0}-L \frac{\dot{u}_{0}(t)}{u_{0}(t)}\right) \xi_{0}(t)+P C^{T}\left(y(t)-C \xi_{0}(t)\right), \\
\dot{v}(t)=\left(A_{0}-L \frac{\dot{u}_{0}(t)}{u_{0}(t)}\right) v(t)+e_{n} u_{1}(t), \\
\dot{P}=P\left(A_{0}-L \frac{\dot{u}_{0}(t)}{u_{0}(t)}\right)^{T}+\left(A_{0}-L \frac{\dot{u}_{0}(t)}{u_{0}(t)}\right) P-P C^{T} C P+I,
\end{gathered}
$$

where $y(t)=z_{1}(t), e_{n}=[0, \ldots, 1]^{T}, \xi_{0}=\left[\xi_{01}, \ldots, \xi_{0 n}\right]^{T}, v=$ $\left[v_{1}, \ldots, v_{n}\right]^{T}, A_{0}=A-K C, C=[1,0, \ldots, 0], K=$ $\left[k_{1}, \ldots, k_{n}\right]^{T}$, and $k_{i}(1 \leq i \leq n)$ are design parameters to be determined later. Let $\widehat{z}(t)=\xi_{0}(t)+\bar{d}_{n} v, \sigma(t)=z(t)-\bar{d}_{n} v(t)$; then, the estimation error $\varepsilon(t)=z(t)-\widehat{z}(t)$ and the newly defined parameter $\sigma(t)$ satisfy the dynamical equations

$$
\begin{aligned}
\dot{\varepsilon}(t)= & \left(A_{0}-L \frac{\dot{u}_{0}(t)}{u_{0}(t)}-P C^{T} C\right) \varepsilon(t) \\
& +\left(K-P C^{T}\right) z_{1}(t)+P C^{T} C \sigma(t)+\Psi+\Phi, \\
\dot{\sigma}(t)= & \left(A_{0}-L \frac{\dot{u}_{0}(t)}{u_{0}(t)}\right) \sigma(t)+K z_{1}(t)+\Psi+\Phi .
\end{aligned}
$$

3.3. Control Design. In this section, the intergrator backstepping approach will be used to design the control laws $u_{0}(t)$ and $u_{1}(t)$ subject to $x_{0}\left(t_{0}\right) \neq 0$. The case that the initial condition $x_{0}\left(t_{0}\right)=0$ will be treated in Section 4 .

Step 0. At this step, control law $u_{0}(t)$ will be designed, which is essential to guarantee the effectiveness of the subsequent steps. For the $x_{0}(t)$-subsystem, choose the control $u_{0}(t)$ as follows:

$$
u_{0}(t)=-\lambda_{0} x_{0}(t)-\lambda_{0} x_{0}(t) \bar{\phi}_{0}\left(x_{0}(t)\right),
$$

where $\lambda_{0}$ is a constant satisfying $\lambda_{0} d_{0}>1$. Introduce the Lyapunov function candidate $V_{0}=(1 / 2) x_{0}^{2}(t)$, and the time derivative of $V_{0}$ satisfies

$$
\begin{aligned}
\dot{V}_{0}= & -\lambda_{0} d_{0} x_{0}^{2}(t)-\lambda_{0} d_{0} x_{0}^{2}(t) \bar{\phi}_{0}\left(x_{0}(t)\right) \\
& +x_{0}(t) \phi_{0}\left(t, x_{0}(t)\right) \\
\leq & -\lambda_{0} d_{0} x_{0}^{2}(t) \triangleq-c_{0} x_{0}^{2}(t),
\end{aligned}
$$

where $c_{0}=\lambda_{0} d_{0}>1$. This indicates that $x_{0}(t)$ converges to zero exponentially.
Since $\bar{\phi}_{0}\left(x_{0}(t)\right)$ is a smooth function, then there exist a constant $M_{0}>1$, such that $\left|\bar{\phi}_{0}\left(x_{0}(t)\right)\right| \leq M_{0}$ for $\left|x_{0}(t)\right| \leq 1$. Therefore, the following inequality is true with $\left|x_{0}(t)\right| \leq 1$ :

$$
\dot{V}_{0} \geq-\left(\lambda_{0} d_{0}+\lambda_{0} d_{0} M_{0}+M_{0}\right) x_{0}^{2}(t) \triangleq-\rho x_{0}^{2}(t)
$$

which implies that when $\left|x_{0}(t)\right| \leq 1$, the state $x_{0}(t)$ converges to zero with a rate less than a certain constant $\rho$. It is $x_{0}(t)$ which does not become zero in any time instant. Therefore, the adopted input-state scaling discontinuous transformation in (12) is effective.

According to the design of control law $u_{0}(t)$ in (23), it can be computed that

$$
\begin{aligned}
\frac{\dot{u}_{0}(t)}{u_{0}(t)}= & -\lambda_{0} d_{0}-\left(\lambda_{0} d_{0}-1\right) \bar{\phi}_{0}\left(x_{0}(t)\right) \\
& -\lambda_{0} d_{0} x_{0}(t) \frac{\partial \bar{\phi}_{0}\left(x_{0}(t)\right)}{\partial x_{0}(t)} \\
& +\frac{x_{0}(t) \bar{\phi}_{0}\left(x_{0}(t)\right)}{1+\bar{\phi}_{0}\left(x_{0}(t)\right)} \frac{\partial \bar{\phi}_{0}\left(x_{0}(t)\right)}{\partial x_{0}(t)} \\
\triangleq & +\widetilde{\phi}_{0}\left(x_{0}(t)\right),
\end{aligned}
$$

where $\beta=-\lambda_{0} d_{0}$ and $\tilde{\phi}_{0}=-\left(\lambda_{0} d_{0}-1\right) \bar{\phi}_{0}\left(x_{0}(t)\right)-$ $\lambda_{0} d_{0} x_{0}(t)\left(\partial \bar{\phi}_{0}\left(x_{0}(t)\right) / \partial x_{0}(t)\right)+\left(x_{0}(t) \bar{\phi}_{0}\left(x_{0}(t)\right) /(1+\right.$ $\left.\left.\bar{\phi}_{0}\left(x_{0}(t)\right)\right)\right)\left(\partial \bar{\phi}_{0}\left(x_{0}(t)\right) / \partial x_{0}(t)\right)$.

Remark 11. From (26), we know that $\beta$ is a constant and $\widetilde{\phi}_{0}\left(x_{0}(t)\right)$ is a function with respect to $x_{0}(t)$. Moreover, we can conclude that $\widetilde{\phi}_{0}\left(x_{0}(t)\right)$ is smooth because $\bar{\phi}_{0}\left(x_{0}(t)\right)$ is a nonnegative smooth function.

Denote $A_{1}=A_{0}-K C-L \beta$; we can choose appropriate design parameters $k_{i}(1 \leq i \leq n)$ such that $A_{1}$ is Hurwitz. Then there exists a positive definite matrix $Q$ satisfying $Q A_{1}+$ $A_{1}^{T} Q=-\mu I$, and $\mu$ is a positive constant.

Step 1. For $z_{1}(t)$-subsystem in (13),

$$
\begin{aligned}
\dot{z}_{1}(t)= & z_{2}(t)-(n-1) \frac{\dot{u}_{0}(t)}{u_{0}(t)} z_{1}(t) \\
& +\frac{1}{u_{0}^{n-1}(t)}\left(\varphi_{1}+\phi_{1}\right) \\
= & \varepsilon_{2}(t)+\xi_{02}(t)+\bar{d}_{n} v_{2}(t) \\
& -(n-1) \frac{\dot{u}_{0}(t)}{u_{0}(t)} z_{1}(t)+\Psi_{1}+\Phi_{1},
\end{aligned}
$$

let $\eta_{1}(t)=z_{1}(t)$, and $\eta_{2}(t)=v_{2}(t)-\alpha_{1}$. Introduce the following Lyapunov functional:

$$
V_{1}=\bar{V}_{1}+\widetilde{V}_{1}
$$


where

$$
\begin{aligned}
\bar{V}_{1}= & \varepsilon^{T}(t) P^{-1} \varepsilon(t)+\sigma^{T}(t) Q \sigma(t) \\
& +\frac{1}{2} \eta_{1}^{2}(t)+\frac{\left|\bar{d}_{n}\right|}{2} \widetilde{\Theta}_{1}^{T} \widetilde{\Theta}_{1} \\
\widetilde{V}_{1}= & \left(4 \ell_{1}+\delta_{2}\|Q\|^{2}\right) \sum_{j=1}^{n} \int_{t-\tau_{j}}^{t} \eta_{1}^{4}(\sigma) f_{j 2}^{4}\left(u_{0}(\sigma), y(\sigma)\right) d \sigma \\
& +\frac{n}{2} \int_{t-\tau_{1}}^{t} \eta_{1}^{2}(\sigma) f_{12}^{2}\left(u_{0}(\sigma), y(\sigma)\right) d \sigma,
\end{aligned}
$$

with $\ell_{1}, \delta_{2}$ being positive constants to be designed; $\widetilde{\Theta}_{1}=\Theta_{1}-$ $\widehat{\Theta}_{1}$, where $\Theta_{1}$ is an unknown parameter vector to be specified later, and $\widehat{\Theta}_{1}$ is an estimate of $\Theta_{1}$.

Associated with (22) and (27), the time derivatives of $\bar{V}_{1}$ and $\widetilde{V}_{1}$ can be calculated, respectively, that

$$
\begin{aligned}
& \dot{\bar{V}}_{1}=2 \varepsilon^{T}(t) P^{-1}\left(A_{0}-L \frac{\dot{u}_{0}(t)}{u_{0}(t)}-P C^{T} C\right) \varepsilon(t) \\
& +2 \varepsilon^{T}(t) P^{-1}\left(K-P C^{T}\right) z_{1}(t) \\
& +2 \varepsilon^{T}(t) C^{T} C \sigma(t)+2 \varepsilon^{T}(t) P^{-1} \Psi \\
& +2 \varepsilon^{T}(t) P^{-1} \Phi+2 \sigma^{T}(t) Q\left(A_{0}-L \frac{\dot{u}_{0}(t)}{u_{0}(t)}\right) \sigma(t) \\
& +2 \sigma^{T}(t) Q K z_{1}(t)+2 \sigma^{T}(t) Q \Psi \\
& +2 \sigma^{T}(t) Q \Phi-2 \varepsilon^{T}(t)\left(A_{0}-L \frac{\dot{u}_{0}(t)}{u_{0}(t)}\right)^{T} P^{-1} \varepsilon(t) \\
& +2 \varepsilon^{T}(t) C^{T} C \varepsilon(t)-\varepsilon^{T}(t) P^{-2} \varepsilon(t) \\
& +\eta_{1}(t)\left[\varepsilon_{2}(t)+\xi_{02}(t)+\bar{d}_{n} v_{2}(t)-(n-1) \frac{\dot{u}_{0}(t)}{u_{0}(t)} z_{1}(t)\right. \\
& \left.+\Psi_{1}+\Phi_{1}\right]-\left|\bar{d}_{n}\right| \widetilde{\Theta}_{1}^{T} \dot{\bar{\Theta}}_{1} \\
& =-\varepsilon^{T}(t) P^{-2} \varepsilon(t)-\mu \sigma^{T}(t) \sigma(t) \\
& +2 \varepsilon^{T}(t) P^{-1}\left(K-P C^{T}\right) z_{1}(t)+2 \varepsilon^{T}(t) P^{-1} \Psi \\
& +2 \varepsilon^{T}(t) P^{-1} \Phi+2 \varepsilon^{T}(t) C^{T} C \sigma(t) \\
& -2 \sigma^{T}(t) Q L \tilde{\phi}\left(x_{0}(t)\right) \sigma(t)+2 \sigma^{T}(t) Q K z_{1}(t) \\
& +2 \sigma^{T}(t) Q \Psi+2 \sigma^{T}(t) Q \Phi+\eta_{1}(t) \Psi_{1}+\eta_{1}(t) \Phi_{1} \\
& -(n-1) \frac{\dot{u}_{0}(t)}{u_{0}(t)} \eta_{1}^{2}(t)+\eta_{1}(t) \varepsilon_{2}(t)-\varepsilon^{T}(t) C^{T} C \varepsilon(t) \\
& +\eta_{1}(t)\left[\xi_{02}(t)+\bar{d}_{n} v_{2}(t)\right]-\left|\bar{d}_{n}\right| \widetilde{\Theta}_{1}^{T} \dot{\Theta}_{1},
\end{aligned}
$$

$$
\begin{aligned}
\dot{\widetilde{V}}_{1}= & \left(4 \ell_{1}+\delta_{2}\|Q\|^{2}\right) \sum_{j=1}^{n} \eta_{1}^{4}(t) f_{j 2}^{4}\left(u_{0}(t), y(t)\right)+\frac{n}{2} \eta_{1}^{2}(t) \\
& \times f_{12}^{2}\left(u_{0}(t), y(t)\right)-\left(4 \ell_{1}+\delta_{2}\|Q\|^{2}\right) \sum_{j=1}^{n} \eta_{1}^{4}\left(t-\tau_{j}\right) \\
& \times f_{j 2}^{4}\left(u_{0}\left(t-\tau_{j}\right), y\left(t-\tau_{j}\right)\right)-\frac{n}{2} \eta_{1}^{2}\left(t-\tau_{1}\right) \\
& \times f_{12}^{2}\left(u_{0}\left(t-\tau_{1}\right), y\left(t-\tau_{1}\right)\right) .
\end{aligned}
$$

For some terms on the right-hand side of (30), the following estimations (32)-(34) should be conducted. Firstly, by Lemma 8 and Young's inequality, we can obtain that there exist positive constants $\ell_{1}, \delta_{1}$ to make the following inequalities hold:

$$
\begin{aligned}
\eta_{1}(t) \Phi_{1} \leq & \eta_{1}^{2}(t)+\frac{1}{4} \eta_{1}^{2}(t) \widetilde{\omega}_{1}^{2}\left(u_{0}(t), x_{0}(t), z_{1}(t)\right) \vartheta^{2} \\
\leq & \eta_{1}^{2}(t)+\frac{1}{4} \eta_{1}^{2}(t) \widetilde{\omega}_{1}^{2}\left(u_{0}(t), x_{0}(t), z_{1}(t)\right) \vartheta_{1}, \\
2 \varepsilon^{T}(t) P^{-1} \Phi \leq & \frac{1}{4 \ell_{1}} \varepsilon^{T}(t) P^{-2} \varepsilon(t) \\
& +4 \ell_{1} \sum_{j=1}^{n} \eta_{1}^{2}(t) \widetilde{\omega}_{j}^{2}\left(u_{0}(t), x_{0}(t), z_{1}(t)\right) \bar{d}_{j-1}^{2} \vartheta^{2} \\
\leq & \frac{1}{4 \ell_{1}} \varepsilon^{T}(t) P^{-2} \varepsilon(t)+4 \ell_{1} \sum_{j=1}^{n} \eta_{1}^{2}(t) \\
& \times \widetilde{\omega}_{j}^{2}\left(u_{0}(t), x_{0}(t), z_{1}(t)\right) \vartheta_{1}, \\
2 \sigma^{T}(t) Q \Phi \leq & \frac{1}{\delta_{1}} \sigma^{T}(t) \sigma(t) \\
& +\delta_{1}\|Q\|^{2} \sum_{j=1}^{n} \eta_{1}^{2}(t) \widetilde{\omega}_{j}^{2}\left(u_{0}(t), x_{0}(t), z_{1}(t)\right) \vartheta_{1},
\end{aligned}
$$

where $\vartheta_{1}=\vartheta^{2}+\sum_{j=1}^{n-1} \bar{d}_{j}^{2} \vartheta^{2}$. Next, employ Lemma 9 and Young's inequality, and we have

$$
\begin{aligned}
\eta_{1}(t) & \Psi_{1} \\
\leq & \eta_{1}^{2}(t) \widetilde{\psi}_{1}\left(u_{0}(t), y(t)\right)+\frac{1}{2} \eta_{1}^{4}(t) f_{11}^{2}\left(u_{0}(t), y(t)\right) \\
& \quad+\frac{1}{2} \eta_{1}^{2}\left(t-\tau_{1}\right) f_{12}^{2}\left(u_{0}\left(t-\tau_{1}\right), y\left(t-\tau_{1}\right)\right), \\
2 \varepsilon^{T}(t) P^{-1} \Psi & \\
\leq & \frac{1}{4 \ell_{1}} \varepsilon^{T}(t) P^{-2} \varepsilon(t)+4 \ell_{1} \sum_{j=1}^{n} \Psi_{j}^{2} \\
& \leq \frac{1}{4 \ell_{1}} \varepsilon^{T}(t) P^{-2} \varepsilon(t)
\end{aligned}
$$




$$
\begin{aligned}
& +8 \ell_{1} \sum_{j=1}^{n} \eta_{1}^{2}(t) \widetilde{\psi}_{j}^{2}\left(u_{0}(t), y(t)\right) \bar{d}_{j-1}^{2} \\
& +4 \ell_{1} \sum_{j=1}^{n} \eta_{1}^{4}\left(t-\tau_{j}\right) f_{j 2}^{4}\left(u_{0}\left(t-\tau_{j}\right), y\left(t-\tau_{j}\right)\right) \\
& +4 \ell_{1} \sum_{j=1}^{n} \eta_{1}^{4}(t) f_{j 1}^{4}\left(u_{0}(t), y(t)\right) \bar{d}_{j-1}^{4} \\
& \leq \frac{1}{4 \ell_{1}} \varepsilon^{T}(t) P^{-2} \varepsilon(t) \\
& \quad+8 \ell_{1} \sum_{j=1}^{n} \eta_{1}^{2}(t) \widetilde{\psi}_{j}^{2}\left(u_{0}(t), y(t)\right) d \\
& \quad+4 \ell_{1} \sum_{j=1}^{n} \eta_{1}^{4}\left(t-\tau_{j}\right) f_{j 2}^{4}\left(u_{0}\left(t-\tau_{j}\right), y\left(t-\tau_{j}\right)\right) \\
& \quad+4 \ell_{1} \sum_{j=1}^{n} \eta_{1}^{4}(t) f_{j 1}^{4}\left(u_{0}(t), y(t)\right) d, \\
& 2 \sigma^{T}(t) Q \Psi \\
& \leq \frac{1}{\delta_{2}} \sigma^{T}(t) \sigma(t)+2 \delta_{2}\|Q\|^{2} \sum_{j=1}^{n} \eta_{1}^{2}(t) \widetilde{\psi}_{j}^{2}\left(u_{0}(t), y(t)\right) d \\
& \quad+\delta_{2}\|Q\|^{2} \sum_{j=1}^{n} \eta_{1}^{4}\left(t-\tau_{j}\right) f_{j 2}^{4}\left(u_{0}\left(t-\tau_{j}\right), y\left(t-\tau_{j}\right)\right) \\
& \quad \delta_{2}\|Q\|^{2} \sum_{j=1}^{n} \eta_{1}^{4}(t) f_{j 1}^{4}\left(u_{0}(t), y(t)\right) d,
\end{aligned}
$$

where $d=1+\sum_{j=1}^{n-1} \bar{d}_{j}^{2}+\sum_{j=1}^{n-1} \bar{d}_{j}^{4}$, and $\delta_{2}$ is a positive constant.

By completing the square, the following estimations are also true:

$$
\begin{gathered}
\eta_{1}(t) \varepsilon_{2}(t) \leq \frac{1}{4 \ell_{1}} \varepsilon^{T}(t) P^{-2} \varepsilon(t)+\ell_{1} P_{\max }^{2} \eta_{1}^{2}(t), \\
2 \varepsilon^{T}(t) P^{-1} K z_{1}(t) \leq \frac{1}{4 \ell_{1}} \varepsilon^{T}(t) P^{-2} \varepsilon(t)+4 \ell_{1} K^{T} K \eta_{1}^{2}(t), \\
-2 \varepsilon^{T}(t) C^{T} z_{1}(t) \leq \frac{1}{2} \varepsilon^{T}(t) C^{T} C \varepsilon(t)+2 \eta_{1}^{2}(t), \\
2 \varepsilon^{T}(t) C^{T} C \sigma(t) \leq \frac{1}{2} \varepsilon^{T}(t) C^{T} C \varepsilon(t)+2 \sigma^{T}(t) \sigma(t), \\
2 \sigma^{T}(t) Q K z_{1}(t) \leq \sigma^{T}(t) \sigma(t)+K^{T} Q^{T} Q K \eta_{1}^{2}(t) .
\end{gathered}
$$

Substitute (31)-(34) into $\dot{V}_{1}$, it yields

$$
\begin{aligned}
\dot{V}_{1}= & \dot{\bar{V}}_{1}+\dot{\vec{V}}_{1} \\
\leq & -\left(1-\frac{1}{\ell_{1}}\right) \varepsilon^{T}(t) P^{-2} \varepsilon(t) \\
& -\bar{c}_{1} \eta_{1}^{2}(t)-(n-1) \widetilde{\phi}\left(x_{0}(t)\right) \eta_{1}^{2}(t) \\
& -\bar{\mu}^{T}(t) \sigma(t)-\widetilde{\phi}\left(x_{0}(t)\right) \sigma^{T}(t)[Q L+L Q] \sigma(t) \\
& -\left|\bar{d}_{n}\right| \widetilde{\Theta}_{1}^{T} \dot{\Theta}_{1} \\
& -\frac{n-1}{2} \eta_{1}^{2}\left(t-\tau_{1}\right) f_{12}^{2}\left(u_{0}\left(t-\tau_{1}\right), y\left(t-\tau_{1}\right)\right) \\
& +\bar{d}_{n} \eta_{1}(t)\left[\Theta_{1}^{T} \Upsilon_{1}+v_{2}(t)\right],
\end{aligned}
$$

where $\bar{\mu}=\mu-1 / \delta_{1}-1 / \delta_{2}-3, \bar{c}_{1}=c_{1}-3-K^{T} Q^{T} Q K-$ $4 \ell_{1} K^{T} K-\ell_{1} P_{\max }^{2}+(n-1) \beta, \Theta_{1}^{T}=\left(1 / \bar{d}_{n}\right)\left[1, d, \vartheta_{1}\right]$, and $\Upsilon_{1}=$ $\left[\Upsilon_{11}, \Upsilon_{12}, \Upsilon_{13}\right]^{T}$ with

$$
\begin{array}{rl}
\Upsilon_{11}=c_{1} & \eta_{1}(t)+\xi_{02}(t) \\
& +\eta_{1}(t) \widetilde{\psi}_{1}\left(u_{0}(t), y(t)\right)+\frac{1}{2} \eta_{1}^{3}(t) f_{11}^{2}\left(u_{0}(t), y(t)\right) \\
& +\left(4 \ell_{1}+\delta_{2}\|Q\|^{2}\right) \sum_{j=1}^{n} \eta_{1}^{3}(t) f_{j 2}^{4}\left(u_{0}(t), y(t)\right) \\
& +\frac{n}{2} \eta_{1}(t) f_{12}^{2}\left(u_{0}(t), y(t)\right), \\
\Upsilon_{12}=8 & 8 \ell_{1} \sum_{j=1}^{n} \eta_{1}(t) \widetilde{\psi}_{j}^{2}\left(u_{0}(t), y(t)\right) \\
& +\left(4 \ell_{1}+\delta_{2}\|Q\|^{2}\right) \sum_{j=1}^{n} \eta_{1}^{3}(t) f_{j 1}^{4}\left(u_{0}(t), y(t)\right), \\
\Upsilon_{13}=( & \left.4 \ell_{1}+\delta_{1}\|Q\|^{2}\right) \sum_{j=1}^{n} \eta_{1}(t) \widetilde{\omega}_{j}^{2}\left(u_{0}(t), x_{0}(t), z_{1}(t)\right) \\
& +\frac{1}{4} \eta_{1}(t) \widetilde{\omega}_{1}^{2}\left(u_{0}(t), x_{0}(t), z_{1}(t)\right) .
\end{array}
$$

Choose the virtual control function $\alpha_{1}$ and the adaptation law of $\widehat{\Theta}_{1}$ as follows:

$$
\begin{gathered}
\alpha_{1}=-\widehat{\Theta}_{1}^{T} \Upsilon_{1}, \\
\dot{\widehat{\Theta}}_{1}=\operatorname{sign}\left(\bar{d}_{n}\right) \Upsilon_{1} \eta_{1}(t) .
\end{gathered}
$$


Notice that $\bar{d}_{n} \eta_{1}(t) \eta_{2}(t) \leq \eta_{1}^{2}(t)+\left(\bar{d}_{n}^{2} / 4\right) \eta_{2}^{2}(t)$, then it follows from (35)-(38) that

$$
\begin{aligned}
\dot{V}_{1} \leq & -\left(1-\frac{1}{\ell_{1}}\right) \varepsilon^{T}(t) P^{-2} \varepsilon(t)-\bar{\mu} \sigma^{T}(t) \sigma(t) \\
& -\left(\bar{c}_{1}-1\right) \eta_{1}^{2}(t)-(n-1) \widetilde{\phi}\left(x_{0}(t)\right) \eta_{1}^{2}(t) \\
& -\widetilde{\phi}\left(x_{0}(t)\right) \sigma^{T}(t)[Q L+L Q] \sigma(t)-\frac{n-1}{2} \\
& \times \eta_{1}^{2}\left(t-\tau_{1}\right) f_{12}^{2}\left(u_{0}\left(t-\tau_{1}\right), y\left(t-\tau_{1}\right)\right)+\frac{\bar{d}_{n}^{2}}{4} \eta_{2}^{2}(t) .
\end{aligned}
$$

Step 2. Introduce the new variable $\eta_{3}(t)=v_{3}(t)-\alpha_{2}$, where $\alpha_{2}$ is regarded as the virtual control input, and take the Lyapunov functional as

$$
V_{2}=V_{1}+\frac{1}{2} \eta_{2}^{2}(t)+\frac{1}{2} \widetilde{\Theta}_{2}^{T} \widetilde{\Theta}_{2}
$$

where $\widetilde{\Theta}_{2}=\Theta_{2}-\widehat{\Theta}_{2}, \Theta_{2}$ is an unknown parameter vector to be defined later, and $\widehat{\Theta}_{2}$ is an estimate of $\Theta_{2}$. Then, combined with (20), (37), and (39), we have

$$
\begin{aligned}
\dot{V}_{2}=\dot{V}_{1}+\eta_{2}(t)\{ & -k_{2} v_{1}(t)-(n-2) \beta v_{2}(t) \\
& -(n-2) \widetilde{\phi}_{0}\left(x_{0}(t)\right) v_{2}(t)+\eta_{3}(t)+\alpha_{2} \\
& -\frac{\partial \alpha_{1}}{\partial \widehat{\Theta}_{1}^{T}} \dot{\Theta}_{1}-\frac{\partial \alpha_{1}}{\partial \xi_{02}} \dot{\xi}_{02}-\frac{\partial \alpha_{1}}{\partial x_{0}} \dot{x}_{0}-\frac{\partial \alpha_{1}}{\partial u_{0}} \dot{u}_{0} \\
& -\frac{\partial \alpha_{1}}{\partial z_{1}}\left[\xi_{02}-(n-1) \frac{\dot{u}_{0}(t)}{u_{0}(t)} z_{1}(t)\right] \\
& -\frac{\partial \alpha_{1}}{\partial z_{1}} \varepsilon_{2}(t)-\frac{\partial \alpha_{1}}{\partial z_{1}} \Psi_{1}-\frac{\partial \alpha_{1}}{\partial z_{1}} \Phi_{1} \\
& \left.-\frac{\partial \alpha_{1}}{\partial z_{1}} \bar{d}_{n} v_{2}(t)\right\}-\widetilde{\Theta}_{2}^{T} \dot{\Theta}_{2} .
\end{aligned}
$$

Using Lemmas 8 and 9 and Young's inequality, the following inequalities hold:

$$
\begin{aligned}
& -\frac{\partial \alpha_{1}}{\partial z_{1}} \eta_{2}(t) \Psi_{1} \\
& \leq \frac{1}{2} \eta_{1}^{2}(t)+\frac{1}{2}\left(\frac{\partial \alpha_{1}}{\partial z_{1}}\right)^{2} \widetilde{\psi}_{1}^{2}\left(u_{0}(t), x_{0}(t), z_{1}(t)\right) \eta_{2}^{2}(t) \\
& \quad+\frac{1}{2}\left(\frac{\partial \alpha_{1}}{\partial z_{1}}\right)^{2} \eta_{1}^{2}(t) f_{11}^{2}\left(u_{0}(t), y(t)\right) \eta_{2}^{2}(t) \\
& \quad+\frac{1}{2} \eta_{1}^{2}\left(t-\tau_{1}\right) f_{12}^{2}\left(u_{0}\left(t-\tau_{1}\right), y\left(t-\tau_{1}\right)\right) \\
& -\frac{\partial \alpha_{1}}{\partial z_{1}} \eta_{2}(t) \Phi_{1}
\end{aligned}
$$

$$
\begin{aligned}
& \leq \frac{1}{2} \eta_{1}^{2}(t)+\frac{1}{2}\left(\frac{\partial \alpha_{1}}{\partial z_{1}}\right)^{2} \widetilde{\omega}_{1}^{2}\left(u_{0}(t), x_{0}(t), z_{1}(t)\right) \eta_{2}^{2}(t) \vartheta^{2}, \\
- & \frac{\partial \alpha_{1}}{\partial z_{1}} \eta_{2}(t) \varepsilon_{2}(t) \\
& \leq \frac{1}{\ell_{2}} \varepsilon^{T}(t) P^{-2} \varepsilon(t)+\frac{\ell_{2}}{4} p_{\max }^{2}\left(\frac{\partial \alpha_{1}}{\partial z_{1}}\right)^{2} \eta_{2}^{2}(t) .
\end{aligned}
$$

By the above inequalities, we get

$$
\begin{aligned}
\dot{V}_{2} \leq & -\left(1-\frac{1}{\ell_{1}}-\frac{1}{\ell_{2}}\right) \varepsilon^{T}(t) P^{-2} \varepsilon(t) \\
- & \bar{\mu} \sigma^{T}(t) \sigma(t)-\left(\bar{c}_{1}-2\right) \eta_{1}^{2}(t) \\
- & (n-1) \widetilde{\phi}\left(x_{0}(t)\right) \eta_{1}^{2}(t) \\
- & \widetilde{\phi}\left(x_{0}(t)\right) \sigma^{T}(t)[Q L+L Q] \sigma(t) \\
- & \frac{n-2}{2} \eta_{1}^{2}\left(t-\tau_{1}\right) f_{12}^{2}\left(u_{0}\left(t-\tau_{1}\right), y\left(t-\tau_{1}\right)\right)+\eta_{2}(t) \\
& \times\left\{-k_{2} v_{1}(t)-(n-2) \beta v_{2}(t)-(n-2) \widetilde{\phi}\left(x_{0}(t)\right)\right. \\
& \times v_{2}(t)-\frac{\partial \alpha_{1}}{\partial \widehat{\Theta}_{1}} \dot{\Theta}_{1}-\frac{\partial \alpha_{1}}{\partial \xi_{02}} \dot{\xi}_{02}-\frac{\partial \alpha_{1}}{\partial x_{0}} \dot{x}_{0}+\eta_{3}(t) \\
& -\frac{\partial \alpha_{1}}{\partial u_{0}} \dot{u}_{0}-\frac{\partial \alpha_{1}}{\partial z_{1}}\left[\xi_{02}-(n-1) \frac{\dot{u}_{0}(t)}{u_{0}(t)} z_{1}(t)\right] \\
& +\frac{1}{2}\left(\frac{\partial \alpha_{1}}{\partial z_{1}}\right)^{2} \eta_{1}^{2}(t) f_{11}^{2}\left(u_{0}(t), y(t)\right) \eta_{2}(t) \\
& +\alpha_{2}+\frac{1}{2}\left(\frac{\partial \alpha_{1}}{\partial z_{1}}\right)^{2} \widetilde{\psi}_{1}^{2}\left(u_{0}(t), x_{0}(t), z_{1}(t)\right) \eta_{2}(t) \\
& \left.+\frac{\ell_{2}}{4} p_{\max }^{2}\left(\frac{\partial \alpha_{1}}{\partial z_{1}}\right)^{2} \eta_{2}(t)+\Theta_{2}^{T} \Upsilon_{2}\right\}-\widetilde{\Theta}_{2}^{T} \dot{\Theta}_{2},
\end{aligned}
$$

where $\Theta_{2}^{T}=\left[\vartheta^{2}, \bar{d}_{n}^{2}, \bar{d}_{n}\right]$ and $\Upsilon_{2}=\left[(1 / 2)\left(\partial \alpha_{1} / \partial z_{1}\right)^{2}\right.$ $\left.\widetilde{\omega}_{1}^{2} \eta_{2}(t), \eta_{2}(t) / 4,-\left(\partial \alpha_{1} / \partial z_{1}\right) v_{2}(t)\right]^{T}$. By taking the adaptation law $\dot{\widehat{\Theta}}_{2}=\Upsilon_{2} \eta_{2}(t)$ and the virtual control function $\alpha_{2}$ as

$$
\begin{aligned}
\alpha_{2}= & -c_{2} \eta_{2}(t)+k_{2} v_{1}(t) \\
& +(n-2) \beta v_{2}(t)+(n-2) \widetilde{\phi}\left(x_{0}(t)\right) v_{2}(t) \\
& +\frac{\partial \alpha_{1}}{\partial z_{1}}\left[\xi_{02}-(n-1) \frac{\dot{u}_{0}(t)}{u_{0}(t)} z_{1}(t)\right]+\frac{\partial \alpha_{1}}{\partial x_{0}} \dot{x}_{0} \\
& +\frac{\partial \alpha_{1}}{\partial u_{0}} \dot{u}_{0}+\frac{\partial \alpha_{1}}{\partial \widehat{\Theta}_{1}} \dot{\Theta}_{1}+\frac{\partial \alpha_{1}}{\partial \xi_{02}} \dot{\xi}_{02}
\end{aligned}
$$




$$
\begin{aligned}
& -\frac{1}{2}\left(\frac{\partial \alpha_{1}}{\partial z_{1}}\right)^{2} \eta_{1}^{2}(t) f_{11}^{2}\left(u_{0}(t), y(t)\right) \eta_{2}(t) \\
& -\widehat{\Theta}_{2}^{T} \Upsilon_{2}-\frac{\ell_{2}}{4} p_{\max }^{2}\left(\frac{\partial \alpha_{1}}{\partial z_{1}}\right)^{2} \eta_{2}(t) \\
& -\frac{1}{2}\left(\frac{\partial \alpha_{1}}{\partial z_{1}}\right)^{2} \widetilde{\psi}_{1}^{2}\left(u_{0}(t), x_{0}(t), z_{1}(t)\right) \eta_{2}(t),
\end{aligned}
$$

we can obtain

$$
\begin{aligned}
\dot{V}_{2} \leq & -\left(1-\frac{1}{\ell_{1}}-\frac{1}{\ell_{2}}\right) \varepsilon^{T}(t) P^{-2} \varepsilon(t) \\
& -\bar{\mu} \sigma^{T}(t) \sigma(t)-\left(\bar{c}_{1}-2\right) \eta_{1}^{2}(t)-c_{2} \eta_{2}^{2}(t) \\
& -(n-1) \tilde{\phi}\left(x_{0}(t)\right) \eta_{1}^{2}(t) \\
& -\tilde{\phi}\left(x_{0}(t)\right) \sigma^{T}(t)[Q L+L Q] \sigma(t)+\eta_{2}(t) \eta_{3}(t) \\
& -\frac{n-2}{2} \eta_{1}^{2}\left(t-\tau_{1}\right) f_{12}^{2}\left(u_{0}\left(t-\tau_{1}\right), y\left(t-\tau_{1}\right) .\right.
\end{aligned}
$$

Step 3. Define that $\eta_{4}(t)=v_{4}(t)-\alpha_{3}$, where $\alpha_{3}$ is the virtual control input, and consider the following Lyapunov functional:

$$
V_{3}=V_{2}+\frac{1}{2} \eta_{3}^{2}(t)+\frac{1}{2} \widetilde{\Theta}_{3}^{T} \widetilde{\Theta}_{3}
$$

The time derivative of $V_{3}$ along the estimator system (20) satisfies

$$
\begin{aligned}
\dot{V}_{3}=\dot{V}_{2}+ & \eta_{3}(t) \\
\times\{ & -k_{3} v_{1}(t)-(n-3) \beta v_{3}(t) \\
& -(n-3) \widetilde{\phi}\left(x_{0}(t)\right) v_{3}(t)+\eta_{4}(t) \\
& +\alpha_{3}-\frac{\partial \alpha_{2}}{\partial \widehat{\Theta}_{1}}-\frac{\partial \alpha_{2}}{\partial \widehat{\Theta}_{2}} \dot{\widehat{\Theta}}_{2}-\frac{\partial \alpha_{2}}{\partial \xi_{02}} \dot{\xi}_{02}-\frac{\partial \alpha_{2}}{\partial x_{0}} \dot{x}_{0} \\
& -\frac{\partial \alpha_{2}}{\partial u_{0}} \dot{u}_{0}-\frac{\partial \alpha_{1}}{\partial z_{1}}\left[\xi_{02}-(n-1) \frac{\dot{u}_{0}(t)}{u_{0}(t)} z_{1}(t)\right] \\
& -\frac{\partial \alpha_{2}}{\partial v_{1}} \dot{v}_{1}-\frac{\partial \alpha_{2}}{\partial v_{2}} \dot{v}_{2}-\frac{\partial \alpha_{1}}{\partial z_{1}} \varepsilon_{2}(t)-\frac{\partial \alpha_{2}}{\partial z_{1}} \Psi_{1}-\frac{\partial \alpha_{2}}{\partial z_{1}} \Phi_{1} \\
& \left.-\frac{\partial \alpha_{2}}{\partial z_{1}} \bar{d}_{n} v_{2}(t)\right\}-\widetilde{\Theta}_{3}^{T} \dot{\widehat{\Theta}}_{3} .
\end{aligned}
$$

By similar conduction method in (42), we have

$$
\begin{aligned}
& -\frac{\partial \alpha_{2}}{\partial z_{1}} \eta_{3}(t) \Psi_{1} \\
& \leq \frac{1}{2} \eta_{1}^{2}(t)+\frac{1}{2}\left(\frac{\partial \alpha_{2}}{\partial z_{1}}\right)^{2} \widetilde{\psi}_{1}^{2}\left(u_{0}(t), x_{0}(t), z_{1}(t)\right) \eta_{3}^{2}(t) \\
& \quad+\frac{1}{2}\left(\frac{\partial \alpha_{2}}{\partial z_{1}}\right)^{2} \eta_{1}^{2}(t) f_{11}^{2}\left(u_{0}(t), y(t)\right) \eta_{3}^{2}(t) \\
& \quad+\frac{1}{2} \eta_{1}^{2}\left(t-\tau_{1}\right) f_{12}^{2}\left(u_{0}\left(t-\tau_{1}\right), y\left(t-\tau_{1}\right)\right) \\
& -\frac{\partial \alpha_{2}}{\partial z_{1}} \eta_{3}(t) \Phi_{1} \\
& \leq \frac{1}{2} \eta_{1}^{2}(t)+\frac{1}{2}\left(\frac{\partial \alpha_{2}}{\partial z_{1}}\right)^{2} \widetilde{\omega}_{1}^{2}\left(u_{0}(t), x_{0}(t), z_{1}(t)\right) \eta_{3}^{2}(t) \vartheta^{2} \\
& -\frac{\partial \alpha_{2}}{\partial z_{1}} \eta_{3}(t) \varepsilon_{2}(t) \\
& \leq \frac{1}{\ell_{3}} \varepsilon^{T}(t) P^{-2} \varepsilon(t)+\frac{\ell_{3}}{4} p_{\max }^{2}\left(\frac{\partial \alpha_{2}}{\partial z_{1}}\right)^{2} \eta_{3}^{2}(t)
\end{aligned}
$$

where $\ell_{3}>0$ is a scalar. Based on (48), it yields

$$
\begin{aligned}
\dot{V}_{3} \leq & -\left(1-\frac{1}{\ell_{1}}-\frac{1}{\ell_{2}}-\frac{1}{\ell_{3}}\right) \varepsilon^{T}(t) P^{-2} \varepsilon(t)-\bar{\mu} \sigma^{T}(t) \sigma(t) \\
- & \left(\bar{c}_{1}-3\right) \eta_{1}^{2}(t)-(n-1) \tilde{\phi}\left(x_{0}(t)\right) \eta_{1}^{2}(t) \\
& -\widetilde{\phi}\left(x_{0}(t)\right) \sigma^{T}(t)[Q L+L Q] \sigma(t) \\
- & \frac{n-3}{2} \eta_{1}^{2}\left(t-\tau_{1}\right) f_{12}^{2}\left(y\left(t-\tau_{1}\right)\right) \\
+ & \eta_{3}(t)\left\{\eta_{2}(t)-k_{3} v_{1}(t)-(n-3) \beta v_{3}(t)-(n-3)\right. \\
& \times \widetilde{\phi}\left(x_{0}(t)\right) v_{3}(t)+\eta_{4}(t)+\alpha_{3}-\frac{\partial \alpha_{2}}{\partial \widehat{\Theta}_{1}} \dot{\Theta}_{1} \\
& -\frac{\partial \alpha_{2}}{\partial \widehat{\Theta}_{2}} \dot{\Theta}_{2}-\frac{\partial \alpha_{1}}{\partial \xi_{02}} \dot{\xi}_{02}-\frac{\partial \alpha_{1}}{\partial x_{0}} \dot{x}_{0}-\frac{\partial \alpha_{1}}{\partial u_{0}} \dot{u}_{0} \\
& -\frac{\partial \alpha_{1}}{\partial z_{1}}\left[\xi_{02}-(n-1) \frac{\dot{u}_{0}(t)}{u_{0}(t)} z_{1}(t)\right] \\
& -\frac{\partial \alpha_{2}}{\partial v_{1}} \dot{v}_{1}-\frac{\partial \alpha_{2}}{\partial v_{2}} \dot{v}_{2}+\frac{\ell_{3}}{4} p_{\max }^{2}\left(\frac{\partial \alpha_{2}}{\partial z_{1}}\right)^{2} \eta_{3}(t) \\
& \left.+\frac{\partial \alpha_{2}}{\partial z_{1}}\right)^{2} \eta_{1}^{2}(t) f_{11}^{2}\left(u_{0}(t), y(t)\right) \eta_{3}(t)
\end{aligned}
$$




$$
\begin{aligned}
& +\frac{1}{2}\left(\frac{\partial \alpha_{2}}{\partial z_{1}}\right)^{2} \widetilde{\psi}_{1}^{2}\left(u_{0}(t), x_{0}(t), z_{1}(t)\right) \\
& \left.\times \eta_{3}(t)+\Theta_{3}^{T} \Upsilon_{3}\right\}-\widetilde{\Theta}_{3}^{T} \dot{\widehat{\Theta}}_{3},
\end{aligned}
$$

where $\Theta_{3}^{T}=\left[\vartheta^{2}, \bar{d}_{n}\right]$ and $\Upsilon_{3}=\left[(1 / 2)\left(\partial \alpha_{2} / \partial z_{1}\right)^{2} \widetilde{\omega}_{1}^{2} \eta_{3}(t)\right.$, $\left.-\left(\partial \alpha_{2} / \partial z_{1}\right) v_{2}(t)\right]^{T}$. Choose the tuning function $\pi_{3} \Upsilon_{3} \eta_{3}(t)$, and the virtual control function $\alpha_{3}$ as follows:

$$
\begin{aligned}
\alpha_{3}= & -c_{3} \eta_{3}(t)-\eta_{2}(t)+k_{3} v_{1}(t) \\
& +(n-3) \beta v_{3}(t)+(n-3) \widetilde{\phi}\left(x_{0}(t)\right) v_{3}(t)+\frac{\partial \alpha_{2}}{\partial \widehat{\widehat{\Theta}}_{1}} \dot{\Theta}_{1} \\
& +\frac{\partial \alpha_{2}}{\partial \widehat{\Theta}_{2}} \dot{\widehat{\Theta}}_{2}+\frac{\partial \alpha_{2}}{\partial \xi_{02}} \dot{\xi}_{02}+\frac{\partial \alpha_{2}}{\partial x_{0}} \dot{x}_{0}+\frac{\partial \alpha_{2}}{\partial u_{0}} \dot{u}_{0} \\
& +\frac{\partial \alpha_{2}}{\partial z_{1}}\left[\xi_{02}-(n-1) \frac{\dot{u}_{0}(t)}{u_{0}(t)} z_{1}(t)\right]+\frac{\partial \alpha_{2}}{\partial v_{1}} \dot{v}_{1} \\
& +\frac{\partial \alpha_{2}}{\partial v_{2}} \dot{v}_{2}-\frac{\ell_{3}}{4} p_{\max }^{2}\left(\frac{\partial \alpha_{2}}{\partial z_{1}}\right)^{2} \eta_{3}(t) \\
& -\frac{1}{2}\left(\frac{\partial \alpha_{2}}{\partial z_{1}}\right)^{2} \eta_{1}^{2}(t) f_{11}^{2}\left(u_{0}(t), y(t)\right) \eta_{3}(t)-\widehat{\Theta}_{3}^{T} \Upsilon_{3} \\
& -\frac{1}{2}\left(\frac{\partial \alpha_{2}}{\partial z_{1}}\right)^{2} \widetilde{\psi}_{1}^{2}\left(u_{0}(t), x_{0}(t), z_{1}(t)\right) \eta_{3}(t)
\end{aligned}
$$

Under the virtual control function $\alpha_{3}$ and the tuning function $\pi_{3}$ defined above, the derivative of $V_{3}$ becomes that

$$
\begin{aligned}
\dot{V}_{3} \leq & -\left(1-\frac{1}{\ell_{1}}-\frac{1}{\ell_{2}}-\frac{1}{\ell_{3}}\right) \varepsilon^{T}(t) P^{-2} \varepsilon(t) \\
& -\left(\bar{c}_{1}-3\right) \eta_{1}^{2}(t)-c_{2} \eta_{2}^{2}(t)-c_{3} \eta_{3}^{2}(t) \\
& -\bar{\mu} \sigma^{T}(t) \sigma(t)-\widetilde{\phi}\left(x_{0}(t)\right) \sigma^{T}(t)[Q L+L Q] \sigma(t) \\
& -(n-1) \widetilde{\phi}\left(x_{0}(t)\right) \eta_{1}^{2}(t)+\eta_{3}(t) \eta_{4}(t)-\widetilde{\Theta}_{3}^{T}\left(\dot{\Theta}_{3}-\pi_{3}\right) \\
& -\frac{n-3}{2} \eta_{1}^{2}\left(t-\tau_{1}\right) f_{12}^{2}\left(u_{0}\left(t-\tau_{1}\right), y\left(t-\tau_{1}\right) .\right.
\end{aligned}
$$

Step $i(4 \leq i \leq n)$. Assume that, at Step $i-1$, a virtual control function $\alpha_{i-1}$, a tuning function $\pi_{i-1}$, and a Lyapunov functional $V_{i-1}$ have been designed in such a way that

$$
\begin{aligned}
\dot{V}_{i-1} \leq & -\left(1-\sum_{j=1}^{i-1} \frac{1}{\ell_{j}}\right) \varepsilon^{T}(t) P^{-2} \varepsilon(t) \\
& -\left(\bar{c}_{1}-i+1\right) \eta_{1}^{2}(t)-\sum_{j=2}^{i-1} c_{j} \eta_{j}^{2}(t) \\
& -\bar{\mu} \sigma^{T}(t) \sigma(t)+\eta_{i-1}(t) \eta_{i}(t)-\tilde{\phi}\left(x_{0}(t)\right) \sigma^{T}(t)
\end{aligned}
$$

$$
\begin{aligned}
& \times[Q L+L Q] \sigma(t)-\frac{n-i+1}{2} \\
& \times \eta_{1}^{2}\left(t-\tau_{1}\right) f_{12}^{2}\left(u_{0}\left(t-\tau_{1}\right), y\left(t-\tau_{1}\right)\right) \\
& -\widetilde{\Theta}_{3}^{T}\left(\dot{\Theta}_{3}-\pi_{i-1}\right)-(n-1) \widetilde{\phi}\left(x_{0}(t)\right) \eta_{1}^{2}(t) \\
& -\sum_{j=3}^{i-2} \frac{\partial \alpha_{j}}{\partial \widehat{\Theta}_{3}}\left(\dot{\Theta}_{3}-\pi_{i-1}\right) \eta_{j+1}(t) .
\end{aligned}
$$

Let $\eta_{i+1}(t)=v_{i+1}(t)-\alpha_{i}$, where $\alpha_{i}$ is regarded as the virtual control input, and choose Lyapunov functional as

$$
V_{i}=V_{i-1}+\frac{1}{2} \eta_{i}^{2}(t)
$$

Based on (52), the time derivative of $V_{i}$ satisfies

$$
\begin{aligned}
\dot{V}_{i}=\dot{V}_{i-1}+\eta_{i}(t)\{ & -k_{i} v_{1}(t)-(n-i) \beta v_{i}(t) \\
& -(n-i) \widetilde{\phi}\left(x_{0}(t)\right) v_{i}(t)+\eta_{i+1}(t) \\
& -\frac{\partial \alpha_{i-1}}{\partial \widehat{\Theta}_{1}}-\frac{\partial \alpha_{i-1}}{\partial \widehat{\Theta}_{2}}-\frac{\partial \alpha_{i-1}}{\partial \widehat{\Theta}_{3}} \dot{\Theta}_{3} \\
& -\frac{\partial \alpha_{i-1}}{\partial z_{1}}\left[\xi_{02}-(n-1) \frac{\dot{u}_{0}(t)}{u_{0}(t)} z_{1}(t)\right] \\
& +\alpha_{i}-\frac{\partial \alpha_{i-1}}{\partial u_{0}} \dot{u}_{0}-\frac{\partial \alpha_{i-1}}{\partial \xi_{02}} \dot{\xi}_{02} \\
& -\sum_{j=1}^{i-1} \frac{\partial \alpha_{i-1}}{\partial v_{j}} \dot{v}_{j}-\frac{\partial \alpha_{i-1}}{\partial z_{1}} \varepsilon_{2}(t)-\frac{\partial \alpha_{i-1}}{\partial z_{1}} \Psi_{1} \\
& \left.-\frac{\partial \alpha_{i-1}}{\partial x_{0}} \dot{x}_{0}-\frac{\partial \alpha_{i-1}}{\partial z_{1}} \Phi_{1}-\frac{\partial \alpha_{i-1}}{\partial z_{1}} \bar{d}_{n} v_{2}(t)\right\}
\end{aligned}
$$

Next, we estimate the following terms in the right-hand side of (53) by Lemmas 8 and 9 and Young's inequality as follows:

$$
\begin{aligned}
& -\frac{\partial \alpha_{i-1}}{\partial z_{1}} \eta_{i}(t) \Psi_{1} \\
& \leq \frac{1}{2} \eta_{1}^{2}(t)+\frac{1}{2}\left(\frac{\partial \alpha_{i-1}}{\partial z_{1}}\right)^{2} \widetilde{\psi}_{1}^{2}\left(u_{0}(t), x_{0}(t), z_{1}(t)\right) \eta_{i}^{2}(t) \\
& \quad+\frac{1}{2}\left(\frac{\partial \alpha_{i-1}}{\partial z_{1}}\right)^{2} \eta_{1}^{2}(t) f_{11}^{2}\left(u_{0}(t), y(t)\right) \eta_{i}^{2}(t) \\
& \quad+\frac{1}{2} \eta_{1}^{2}\left(t-\tau_{1}\right) f_{12}^{2}\left(u_{0}\left(t-\tau_{1}\right), y\left(t-\tau_{1}\right)\right) \\
& -\frac{\partial \alpha_{i-1}}{\partial z_{1}} \eta_{i}(t) \Phi_{1}
\end{aligned}
$$




$$
\begin{aligned}
& \leq \frac{1}{2} \eta_{1}^{2}(t)+\frac{1}{2}\left(\frac{\partial \alpha_{i-1}}{\partial z_{1}}\right)^{2} \widetilde{\omega}_{1}^{2} \eta_{i}^{2}(t) \vartheta^{2}, \\
- & \frac{\partial \alpha_{i-1}}{\partial z_{1}} \eta_{i}(t) \varepsilon_{2}(t) \\
& \leq \frac{1}{\ell_{i}} \varepsilon^{T}(t) P^{-2} \varepsilon(t)+\frac{\ell_{i}}{4} p_{\max }^{2}\left(\frac{\partial \alpha_{i-1}}{\partial z_{1}}\right)^{2} \eta_{i}^{2}(t) .
\end{aligned}
$$

Choosing the virtual control function $\alpha_{i}$ as

$$
\begin{aligned}
\alpha_{i}= & -c_{i} \eta_{i}(t)-\eta_{i-1}(t)+k_{i} v_{1}(t) \\
& +(n-i) \beta v_{i}(t)+(n-i) \tilde{\phi}\left(x_{0}(t)\right) v_{i}(t) \\
& +\sum_{j=1}^{i-1} \frac{\partial \alpha_{i-1}}{\partial v_{j}} \dot{v}_{j}+\frac{\partial \alpha_{i-1}}{\partial \widehat{\Theta}_{1}} \dot{\Theta}_{1}+\frac{\partial \alpha_{i-1} \widehat{\Theta}_{2}}{\partial \widehat{\Theta}_{2}} \\
& +\frac{\partial \alpha_{i-1}}{\partial x_{0}} \dot{x}_{0}+\sum_{j=3}^{i-2} \frac{\partial \alpha_{j}}{\partial \widehat{\Theta}_{3}} \Upsilon_{i} \eta_{j+1}(t)+\frac{\partial \alpha_{i-1}}{\partial u_{0}} \dot{u}_{0} \\
& +\frac{\partial \alpha_{i-1}}{\partial z_{1}}\left[\xi_{02}-(n-1) \frac{\dot{u}_{0}(t)}{u_{0}(t)} z_{1}(t)\right] \\
& -\frac{\ell_{i}}{4} p_{\max }^{2}\left(\frac{\partial \alpha_{i-1}}{\partial z_{1}}\right)^{2} \eta_{i}(t)+\frac{\partial \alpha_{i-1}}{\partial \widehat{\Theta}_{3}} \pi_{i} \\
& -\frac{1}{2}\left(\frac{\partial \alpha_{i-1}}{\partial z_{1}}\right)^{2} \eta_{1}^{2}(t) f_{11}^{2}(y(t)) \eta_{i}(t)+\frac{\partial \alpha_{i-1}}{\partial \xi_{02}} \dot{\xi}_{02}-\widehat{\Theta}_{3}^{T} \Upsilon_{i},
\end{aligned}
$$

and the tuning function $\pi_{i}=\pi_{i-1}+\Upsilon_{i} \eta_{i}(t)$ with $\Upsilon_{i}=$ $\left[(1 / 2)\left(\partial \alpha_{i-1} / \partial z_{1}\right)^{2} \widetilde{\omega}_{1}^{2} \eta_{i}(t),-\left(\partial \alpha_{i-1} / \partial z_{1}\right) v_{2}(t)\right]^{T}$. Then, we can show that

$$
\begin{aligned}
\dot{V}_{i} \leq & -\left(1-\sum_{j=1}^{i} \frac{1}{\ell_{j}}\right) \varepsilon^{T}(t) P^{-2} \varepsilon(t) \\
& -\left(\bar{c}_{1}-i\right) \eta_{1}^{2}(t)-\sum_{j=2}^{i} c_{j} \eta_{j}^{2}(t) \\
& -\bar{\mu} \sigma^{T}(t) \sigma(t)-\widetilde{\phi}\left(x_{0}(t)\right) \sigma^{T}(t)[Q L+L Q] \sigma(t) \\
& -(n-1) \times \widetilde{\phi}\left(x_{0}(t)\right) \eta_{1}^{2}(t) \\
& -\widetilde{\Theta}_{3}^{T}\left(\dot{\Theta}_{3}-\pi_{i}\right)-\sum_{j=3}^{i-1} \frac{\partial \alpha_{j}}{\partial \widehat{\Theta}_{3}} \eta_{j+1}(t)\left(\dot{\widehat{\Theta}}_{3}-\pi_{i}\right) \\
& -\frac{n-i}{2} \eta_{1}^{2}\left(t-\tau_{1}\right) f_{12}^{2}\left(u_{0}\left(t-\tau_{1}\right), y\left(t-\tau_{1}\right)\right) \\
& +\eta_{i}(t) \eta_{i+1}(t) .
\end{aligned}
$$

At the last step $(i=n)$, the true input $u_{1}(t)$ will be designed on the basis of the virtual control $\alpha_{i}^{\prime} s$ and the Lyapunov function $V_{n-1}$ introduced before.
The actual control input $u_{1}(t)$ can be designed as

$$
\begin{aligned}
u_{1}(t)= & -c_{n} \eta_{n}(t)-\eta_{n-1}(t)+k_{n} v_{1}(t) \\
& +\frac{\partial \alpha_{n-1}}{\partial \widehat{\Theta}_{1}} \dot{\Theta}_{1}+\frac{\partial \alpha_{n-1}}{\partial \widehat{\Theta}_{2}} \dot{\Theta}_{2}+\frac{\partial \alpha_{n-1}}{\partial \xi_{02}} \dot{\xi}_{02} \\
& +\frac{\partial \alpha_{n-1}}{\partial x_{0}} \dot{x}_{0}+\frac{\partial \alpha_{n-1}}{\partial u_{0}} \dot{u}_{0} \\
& +\frac{\partial \alpha_{n-1}}{\partial z_{1}}\left[\xi_{02}-(n-1) \frac{\dot{u}_{0}(t)}{u_{0}(t)} z_{1}(t)\right]-\widehat{\Theta}_{3}^{T} \Upsilon_{n} \\
& +\frac{\partial \alpha_{n-1}}{\partial \widehat{\Theta}_{3}} \pi_{n}-\frac{1}{2}\left(\frac{\partial \alpha_{n-1}}{\partial z_{1}}\right)^{2} \eta_{1}^{2}(t) f_{11}^{2}(y(t)) \eta_{n}(t) \\
& -\frac{\ell_{n}}{4} p_{\max }^{2}\left(\frac{\partial \alpha_{n-1}}{\partial z_{1}}\right)^{2} \eta_{n}(t) \\
& +\sum_{j=1}^{n-1} \frac{\partial \alpha_{n-1}}{\partial v_{j}} \dot{v}_{j}+\sum_{j=3}^{n-2} \frac{\partial \alpha_{j}}{\partial \widehat{\Theta}_{3}} \Upsilon_{n} \eta_{j+1}(t),
\end{aligned}
$$

and the update law $\widehat{\Theta}_{3}=\pi_{n}$ with $\pi_{n}=\pi_{n-1}+\Upsilon_{n} \eta_{n}(t)$ and $\Upsilon_{n}=\left[(1 / 2)\left(\partial \alpha_{n-1} / \partial z_{1}\right)^{2} \widetilde{\omega}_{1}^{2} \eta_{n}(t),-\left(\partial \alpha_{n-1} / \partial z_{1}\right) v_{2}(t)\right]^{T}$. Eventually, it can be achieved that

$$
\begin{aligned}
\dot{V}_{n} \leq & -\left(1-\sum_{j=1}^{n} \frac{1}{\ell_{j}}\right) \varepsilon^{T}(t) P^{-2} \varepsilon(t) \\
& -\bar{\mu} \sigma^{T}(t) \sigma(t)-\left(\bar{c}_{1}-n\right) \eta_{1}^{2}(t)-\sum_{j=2}^{n} c_{j} \eta_{j}^{2}(t)-(n-1) \\
& \times \widetilde{\phi}\left(x_{0}(t)\right) \eta_{1}^{2}(t)-\widetilde{\phi}\left(x_{0}(t)\right) \sigma^{T}(t)[Q L+L Q] \sigma(t) .
\end{aligned}
$$

3.4. Stability Analysis. Notice that $\widetilde{\phi}\left(x_{0}(t)\right)$ tends to zero as $x_{0}(t)$ converges to origin, and $\delta_{1}, \delta_{2}, \ell_{i}, c_{i}(1 \leq i \leq n)$ in (59) are positive design parameters. Therefore, by an appropriate parameter choice, there exist positive constants $\lambda_{i}>0(1 \leq$ $i \leq n+2)$ such that

$$
\begin{aligned}
\dot{V}_{n} \leq & -\sum_{j=1}^{n} \lambda_{j} \eta_{j}^{2}(t)-\lambda_{n+1} \varepsilon^{T}(t) P^{-2} \varepsilon(t) \\
& -\lambda_{n+2} \sigma^{T}(t) \sigma(t) .
\end{aligned}
$$

It can be seen that $\eta_{i}(t), \varepsilon(t), \sigma(t), \widetilde{\Theta}_{1}, \widetilde{\Theta}_{2}, \widetilde{\Theta}_{3}$ are bounded. Since $\theta$ and $d_{i}$ are unknown bounded parameters, $\widehat{\Theta}_{1}, \widehat{\Theta}_{2}, \widehat{\Theta}_{1}$ are bounded. According to estimator equations (19)-(21), it can be deduced that the boundedness of $z_{1}(t)=\eta_{1}(t)$ guarantees the boundedness of $\xi_{0}(t)$, and then $v_{1}(t)=$ $\left(1 / \bar{d}_{n}\right)\left(z_{1}(t)-\sigma_{1}(t)\right)$ and $\alpha_{1}$ are also bounded. By similar analysis, we can conclude that all signals of the closed loop system are bounded.

By LaSalle invariant Theorem, it further achieves that $\eta_{i}(t), \varepsilon(t), \sigma(t), \widetilde{\Theta}_{1}, \widetilde{\Theta}_{2}, \widetilde{\Theta}_{3} \rightarrow 0$ as $t \rightarrow \infty$. By the controller 
design procedure, we get that $\xi_{0}(t), v(t), \alpha_{i}, u_{1}(t)$ asymptotically tend to zero. Then, the definitions $\widehat{z}(t)=\xi_{0}(t)+\bar{d}_{n} v(t)$ and $z(t)=\varepsilon(t)+\widehat{z}(t)$ show the asymptotical convergence of $\widehat{z}(t)$ and $z(t)$. Finally, from the transformations (10) and (12), we know $x_{i}(t)=\left(1 / \bar{d}_{n}\right) u_{0}^{n-i}(t) z_{i}(t)$, which indicates that the states $x_{i}(t)$ asymptotically converge to zero with the initial condition $x_{0}\left(t_{0}\right) \neq 0$.

For purposes of analysis, we can rewrite the system (14) as follows:

$\dot{z}(t)=\left(A_{1}-L \bar{\phi}_{0}\left(x_{0}(t)\right)\right) z(t)+K z_{1}(t)+B u_{1}(t)+\Psi+\Phi$.

To solve the above differential equation, we have $z(t)$

$$
\begin{aligned}
= & e^{\left(A_{1}-L \bar{\phi}_{0}\left(x_{0}(t)\right)\right) t} z\left(t_{0}\right) \\
& +\int_{t_{0}}^{t} e^{\left(A_{1}-L \bar{\phi}_{0}\left(x_{0}(t)\right)\right)(t-s)}\left[K z_{1}(s)+B u_{1}(s)+\Psi+\Phi\right] d s .
\end{aligned}
$$

Notice that $A_{1}=A-K C-L \beta$ is Hurwitz, and $\bar{\phi}_{0}\left(x_{0}(t)\right)$ tends to zero as $x_{0}(t) \rightarrow 0$, then by Lemmas 8 and 9 , there exist constants $\varrho_{1}>0, \varrho_{2}>0$ such that

$$
\begin{aligned}
|z(t)| \leq & \varrho_{1} e^{-\varrho_{2} t}\left|z\left(t_{0}\right)\right| \\
& +\int_{t_{0}}^{t} \varrho_{1} e^{-\varrho_{2}(t-s)}\left[\|K\| \cdot\left|z_{1}(s)\right|+\|B\|\right. \\
\leq & \left.\cdot\left|u_{1}(s)\right|+\|\Psi\|+\|\Phi\|\right] d s \\
& +\varrho_{1} e^{-\varrho_{2} t}\left|z\left(t_{0}\right)\right| \\
& \left.+\left|z_{1}(s)\right| \widetilde{G}_{1}+\left|z_{1}(s)\right| \widetilde{G}_{2}\right] d s,
\end{aligned}
$$

where $\widetilde{G}_{1}$ is a nonnegative smooth function of $d_{i}, u_{0}(s), u_{0}(s-$ $\left.\tau_{i}\right), y(s), y\left(s-\tau_{i}\right)$, and $\widetilde{G}_{2}$ is a nonnegative smooth function of $d_{i}, u_{0}(s), x_{0}(s), z_{1}(s), \vartheta$.

Since $x_{0}(t), x_{1}(t), u_{0}(t)$ and the system parameters are all bounded, then $\widetilde{G}_{1}, \widetilde{G}_{2}$ in (63) are also bounded. Employing the convergence of $x_{0}(t), z_{1}(t), u_{1}(t)$, we can get that $z(t)$ system is globally asymptotically convergent. From the introduced transformations before, it can be deduced that system (1) is also asymptotically convergent. Now, we can express the following theorem.

Theorem 12. For system (1), under Assumptions 1-3, if the control strategies (23) and (58) are applied with an appropriate choice of the design parameters, the global asymptotic stabilization of the closed loop system is achieved for $x_{0}\left(t_{0}\right) \neq 0$.

In the next section, we will deal with the stability analysis of the closed loop as long as the initial condition $x_{0}\left(t_{0}\right)$ is zero.

\section{Switching Controller}

Several switching controllers have been proposed in some existing literatures. As well known, the choice of a constant feedback for $u_{0}(t)$ may lead to a finite escape. In this note, the following switching category can be designed for the stabilization of system (1) with the initial sate $x_{0}\left(t_{0}\right)=0$. Choosing controller $u_{0}(t)$ as

$$
u_{0}(t)=\operatorname{sign}\left(d_{0}\right) u_{0}^{*}, \quad \text { when }\left|x_{0}(t)\right| \leq \varrho_{3}<x_{0}^{*},
$$

where $u_{0}^{*}>0$ and $\varrho_{3}>0$ are constants.

Since $x_{0}\left(t_{0}\right)=0$, then $\dot{x}_{0}\left(t_{0}\right)$ with $u_{0}(t)$ can be deduced

$$
\dot{x}_{0}\left(t_{0}\right)=\left|d_{0}\right| u_{0}^{*}+\phi\left(t, x_{0}\left(t_{0}\right)\right)=\left|d_{0}\right| u_{0}^{*}>0,
$$

then during the initial small time period, $x_{0}(t)$ is increasing and satisfies $\left|x_{0}(t)\right|+\left|x_{0}(t)\right| \bar{\phi}_{0}\left(x_{0}(t)\right)<\left|d_{0}\right| u_{0}^{*}$.

Choose $x_{0}^{*}$ that satisfy

$$
\left|x_{0}^{*}\right|+\left|x_{0}^{*}\right| \bar{\phi}_{0}\left(x_{0}^{*}\right)=\left|d_{0}\right| u_{0}^{*} \text {. }
$$

Obviously, $x_{0}(t)$ is increasing when $x_{0}(t) \leq x_{0}^{*}$. When $\left|x_{0}(t)\right| \leq \varrho<x_{0}^{*}$, choose the controller $u_{0}(t)=\operatorname{sign}\left(d_{0}\right) u_{0}^{*}$, and the controller $u_{1}(t)$ can be designed according to the simple nonlinear backstepping iterative approach. Since $\left|x_{0}(t)\right|>$ $\varrho_{3}$, at $t_{s}$, we switch the control laws $u_{0}(t)$ and $u_{1}(t)$ into (23) and (58), respectively.

Theorem 13. For system (1), under Assumptions 1-3, if above switching control strategy is applied with an appropriate choice of the design parameters, then the closed-loop system is globally asymptotic regulated at the origin for $x_{0}\left(t_{0}\right)=0$.

\section{Simulation Example}

In this section, a numerical example will be given to illustrate that the proposed systematic control law design method is effective. Consider the following system:

$$
\begin{aligned}
\dot{x}_{0}(t)= & d_{0} u_{0}(t)+x_{0}(t)^{3}, \\
\dot{x}_{1}(t)= & d_{1} u_{0}(t) x_{2}(t)+\frac{1}{2} \ln \left(1+x_{1}^{2}(t)\right) e^{x_{0}(t)} \\
& \times x_{1}^{2}(t-0.3)+x_{1}(t) \theta_{1}^{x_{1}(t)}, \\
\dot{x}_{2}(t)= & d_{2} u_{1}(t)+x_{1}(t) e^{x_{0}(t-0.2)} \\
& \times x_{1}^{3}(t-0.2)+\ln \left(1+\left(\theta_{2} x_{2}(t)\right)^{2}\right), \\
y(t)= & {\left[x_{0}(t), x_{1}(t)\right]^{T}, }
\end{aligned}
$$

where $d_{0}, d_{1}, d_{2}$ are virtual control directions with $d_{1}, d_{2}$ unknown and $d_{0}$ known, and the sign of $\bar{d}_{2}=d_{1} d_{2}$ is also known. $\theta_{1}, \theta_{2}$ are unknown bounded parameters. Next, we consider to design the controller $u_{0}(t)$ and $u_{1}(t)$ to asymptotically stabilize system (67) by the measurable 

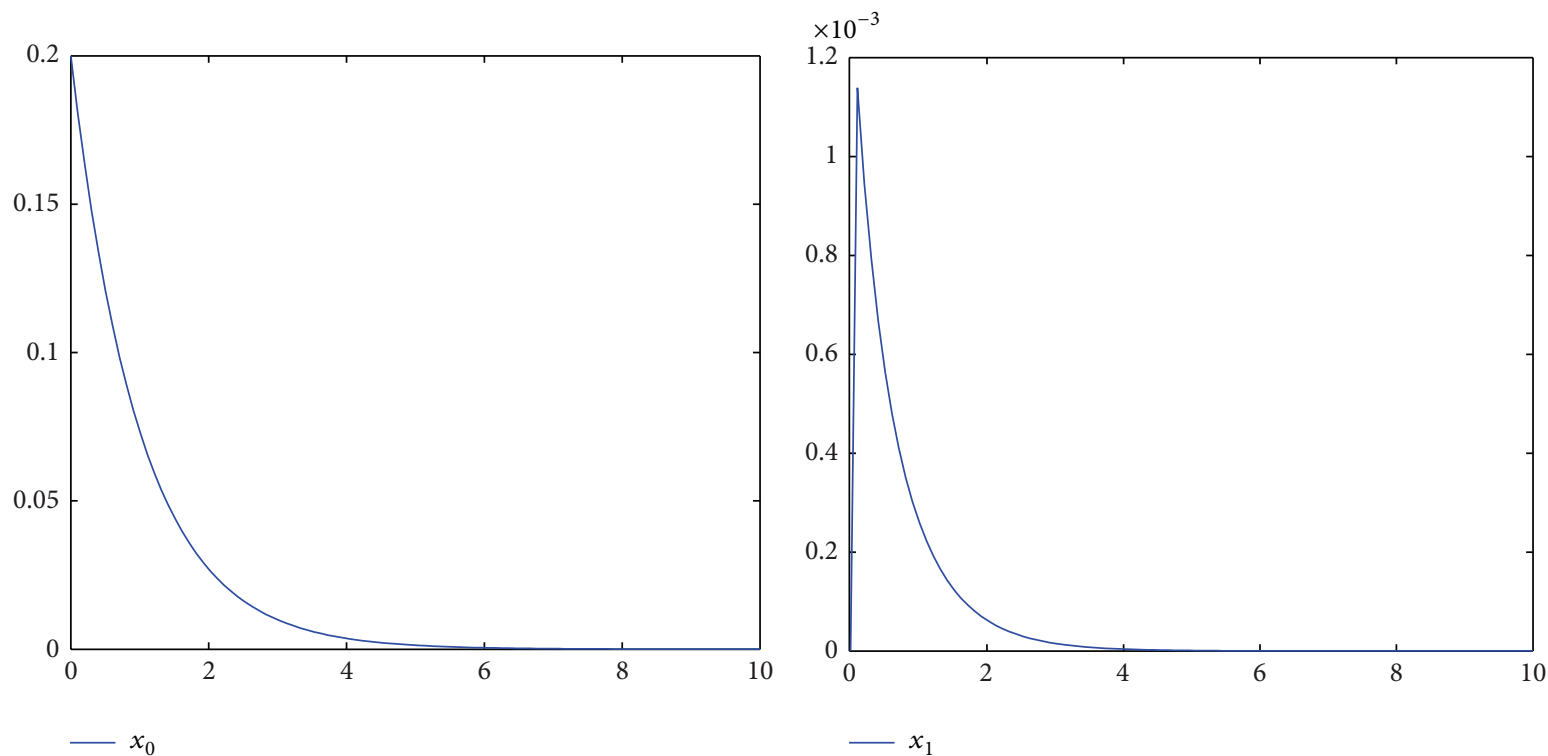

(a)

(b)

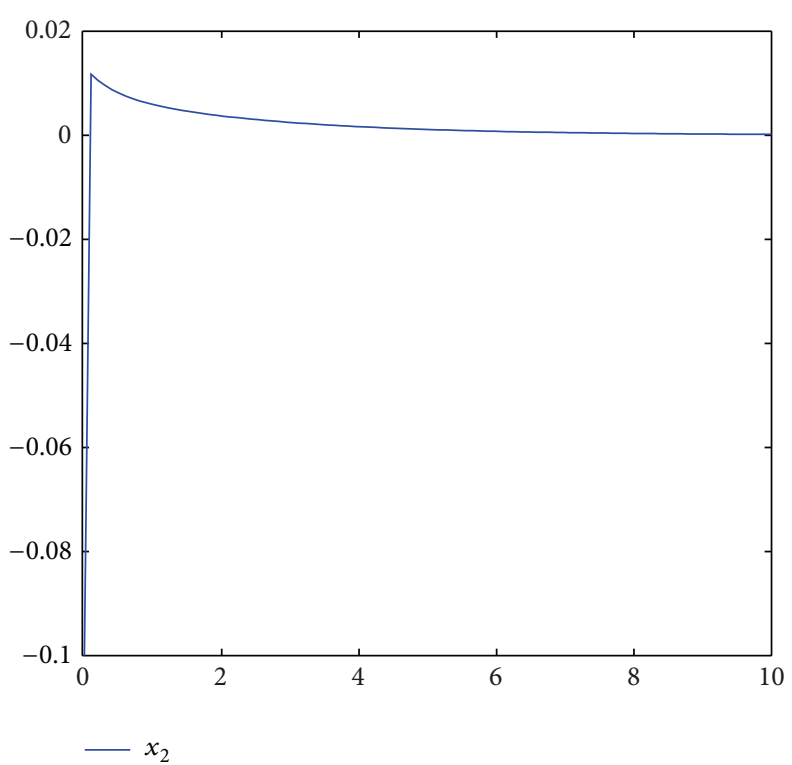

(c)

Figure 1: States $x_{0}(t), x_{1}(t), x_{2}(t)$.

output. We assume that $x_{0}\left(t_{0}\right) \neq 0$ and make the following estimation for some nonlinear terms in system (67):

$$
\begin{aligned}
& x_{1}(t) \theta_{1}^{x_{1}(t)} \leq\left|x_{1}(t)\right| e^{(1 / 2) x_{1}^{2}(t)} \vartheta, \\
& \ln \left(1+\left(\theta_{2} x_{2}(t)\right)^{2}\right) \leq\left|x_{1}(t)\right| \vartheta,
\end{aligned}
$$

where $\vartheta=e^{(1 / 2) \ln ^{2} \theta_{1}}+\left|\theta_{2}\right|$.

Firstly, we introduce the following transformation:

$$
\bar{x}_{1}(t)=x_{1}(t), \quad \bar{x}_{2}(t)=d_{1} x_{2}(t),
$$

and then the system (67) can be rewritten as

$$
\begin{aligned}
\dot{x}_{0}(t)= & d_{0} u_{0}(t)+x_{0}(t)^{3}, \\
\dot{\bar{x}}_{1}(t)= & u_{0}(t) \bar{x}_{2}(t)+\frac{1}{2} \ln \left(1+x_{1}^{2}(t)\right) e^{x_{0}(t)} \\
& \times x_{1}^{2}(t-0.3)+x_{1}(t) \theta_{1}^{x_{1}(t)}, \\
\dot{\bar{x}}_{2}(t)= & \bar{d}_{2} u_{1}(t)+d_{1} x_{1}(t) e^{x_{0}(t-0.2)} \\
& \times x_{1}^{3}(t-0.2)+d_{1} \ln \left(1+\left(\theta_{2} x_{2}(t)\right)^{2}\right),
\end{aligned}
$$

where $\bar{d}_{2}=d_{1} d_{2}$, and assume that the sign of $\bar{d}_{2}$ is known. 


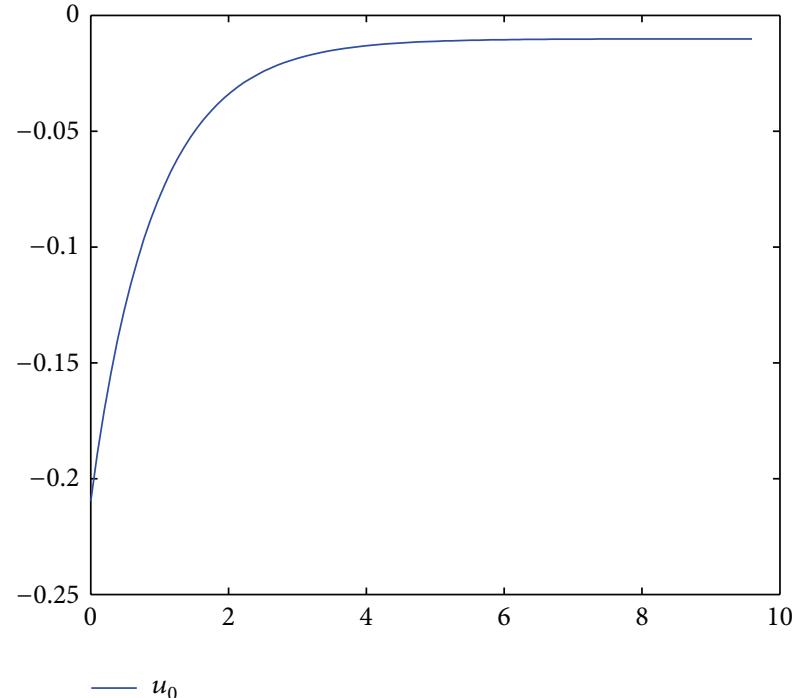

(a)

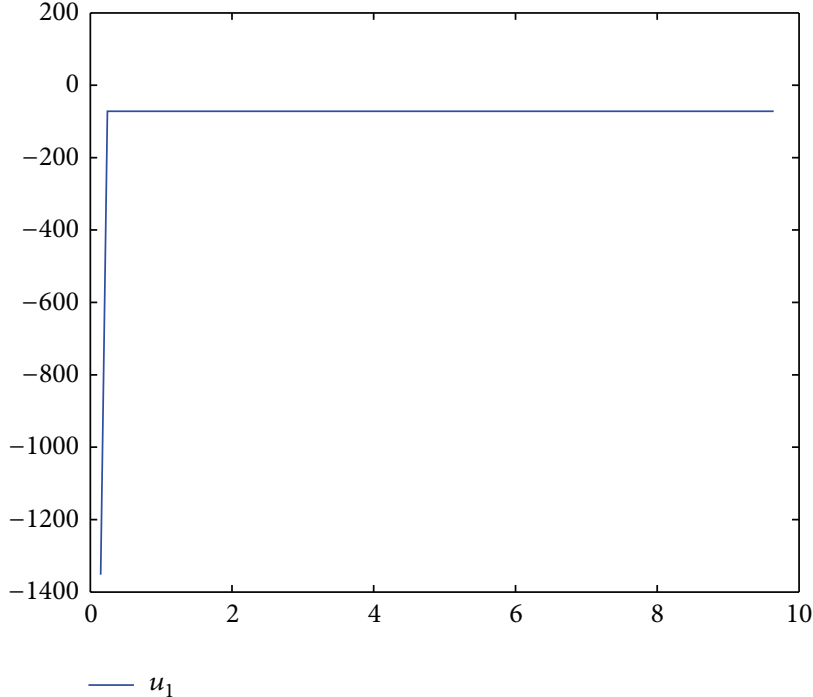

(b)

Figure 2: Controllers $u_{0}(t)$ and $u_{1}(t)$.

Next, make the following input scaling transformation for $\bar{x}(t)$-system:

$$
z_{1}(t)=\frac{\bar{x}_{1}(t)}{u_{0}(t)}, \quad z_{2}(t)=\bar{x}_{2}(t)
$$

and then the transformed system is

$$
\dot{z}(t)=\left(A-L \frac{\dot{u}_{0}(t)}{u_{0}(t)}\right) z(t)+B u_{1}(t)+\Psi+\Phi,
$$

where

$$
\begin{aligned}
& A=\left[\begin{array}{ll}
0 & 1 \\
0 & 0
\end{array}\right], \quad L=\left[\begin{array}{ll}
1 & 0 \\
0 & 0
\end{array}\right], \\
& B=\left[\begin{array}{l}
0 \\
\bar{d}_{2}
\end{array}\right], \quad \Psi=\left[\begin{array}{l}
\Psi_{1} \\
\Psi_{2}
\end{array}\right], \quad \Phi=\left[\begin{array}{l}
\Phi_{1} \\
\Phi_{2}
\end{array}\right], \\
& \Psi_{1}=\frac{\ln \left(1+x_{1}^{2}(t)\right) e^{x_{0}(t)} x_{1}^{2}(t-0.3)}{2 u_{0}(t)}, \\
& \Psi_{i}=d_{1} x_{1}(t) e^{x_{0}(t-0.2)} x_{1}^{3}(t-0.2), \\
& \Phi_{1}=\frac{x_{1}(t) \theta_{1}^{x_{1}(t)}}{u_{0}(t)}, \\
& \Phi_{2}=d_{1} \ln \left(1+\left(\theta_{2} x_{2}(t)\right)^{2}\right) \cdot \frac{\dot{u}_{0}(t)}{u_{0}(t)} .
\end{aligned}
$$

Design the following controller $u_{0}(t)$ :

$$
u_{0}(t)=-c_{0} x_{0}(t)-c_{0} x_{0}(t)^{3},
$$

and then $\dot{u}_{0}(t) / u_{0}(t)$ can be calculated as follows:

$$
\frac{\dot{u}_{0}(t)}{u_{0}(t)}=-c_{0} d_{0}-3 c_{0} d_{0} x_{0}(t)+\frac{x_{0}^{2}(t)+3 x_{0}^{4}(t)}{1+x_{0}^{2}(t)} \text {. }
$$

For system (72), constructing the following estimator:

$$
\begin{aligned}
& \dot{\xi}_{0}(t)=\left(A_{0}-L \frac{\dot{u}_{0}(t)}{u_{0}(t)}\right) \xi_{0}(t)+P C^{T}\left(y(t)-C \xi_{0}(t)\right), \\
& \dot{v}(t)=\left(A_{0}-L \frac{\dot{u}_{0}(t)}{u_{0}(t)}\right) v(t)+e_{n} u_{1}(t), \\
& \dot{P}=P\left(A_{0}-L \frac{\dot{u}_{0}(t)}{u_{0}(t)}\right)^{T}+\left(A_{0}-L \frac{\dot{u}_{0}(t)}{u_{0}(t)}\right) P-P C^{T} C P+I,
\end{aligned}
$$

where $y(t)=z_{1}(t), e_{n}=[0,1]^{T}, \xi_{0}=\left[\xi_{01}, \xi_{02}\right]^{T}, v=\left[v_{1}, v_{2}\right]^{T}$, $A_{0}=A-K C, C=[1,0]$, and $K=\left[k_{1}, k_{2}\right]^{T}$. The design of $k_{1}, k_{2}$ can guarantee that $A_{1}=A_{0}-K C-L \beta$ is Hurwitz. It is further achieved that there exists plosive definite matrix $Q$ satisfying $Q A_{1}+A_{1}^{T} Q=-\mu I$, in which $\mu>0$ is a constant. Denote $\widehat{z}(t)=\xi_{0}(t)+\bar{d}_{n} v, \sigma(t)=z(t)-\bar{d}_{n} v(t)$ and $\varepsilon(t)=$ $z(t)-\widehat{z}(t)$, and then the observation error $\varepsilon(t)$ and parameter invariable $\sigma(t)$ satisfy

$$
\begin{aligned}
\dot{\varepsilon}(t)= & \left(A_{0}-L \frac{\dot{u}_{0}(t)}{u_{0}(t)}-P C^{T} C\right) \varepsilon(t) \\
& +\left(K-P C^{T}\right) z_{1}(t)+P C^{T} C \sigma(t)+\Psi+\Phi, \\
\dot{\sigma}(t)= & \left(A_{0}-L \frac{\dot{u}_{0}(t)}{u_{0}(t)}\right) \sigma(t)+K z_{1}(t)+\Psi+\Phi .
\end{aligned}
$$



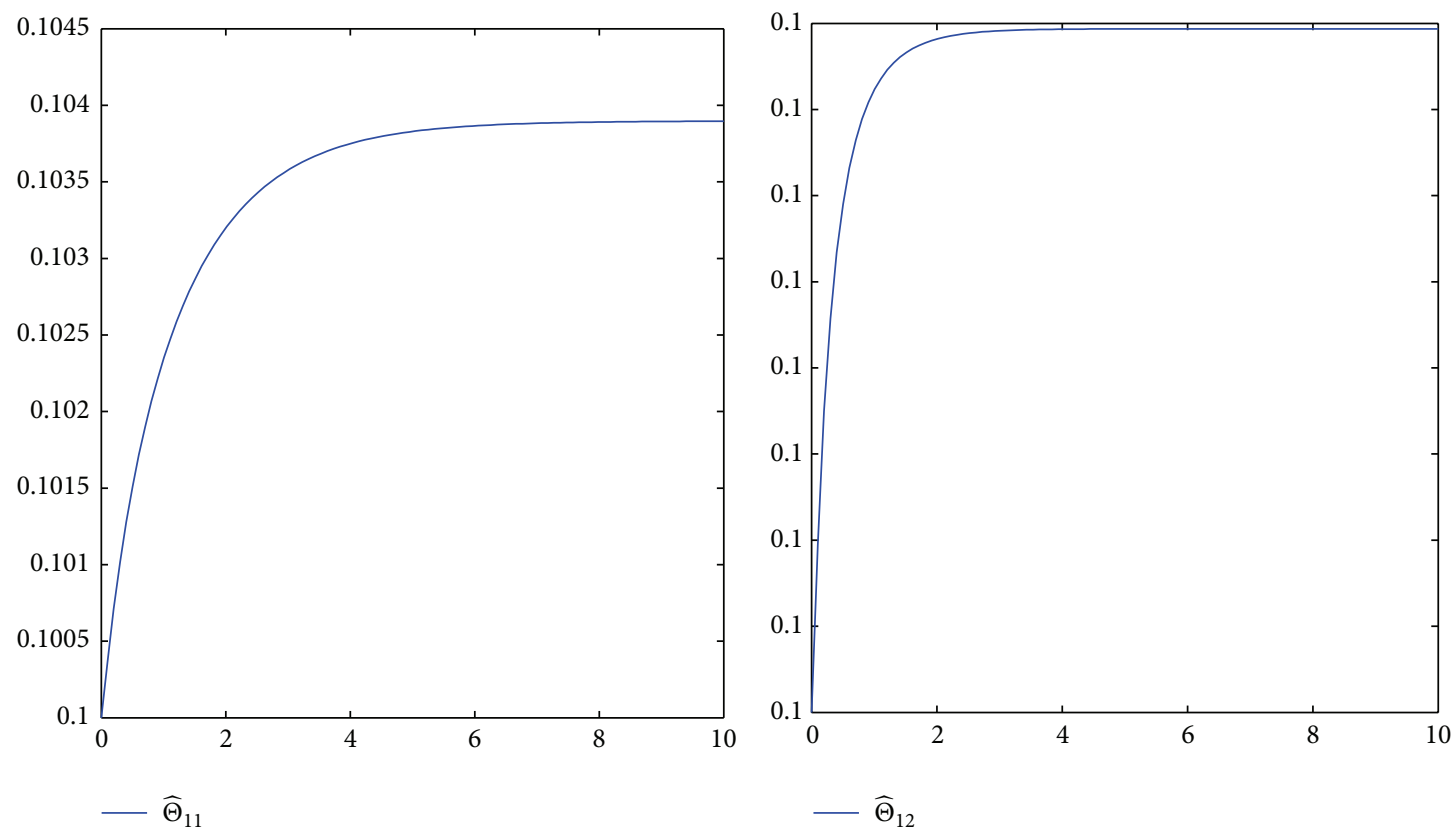

(a)

(b)

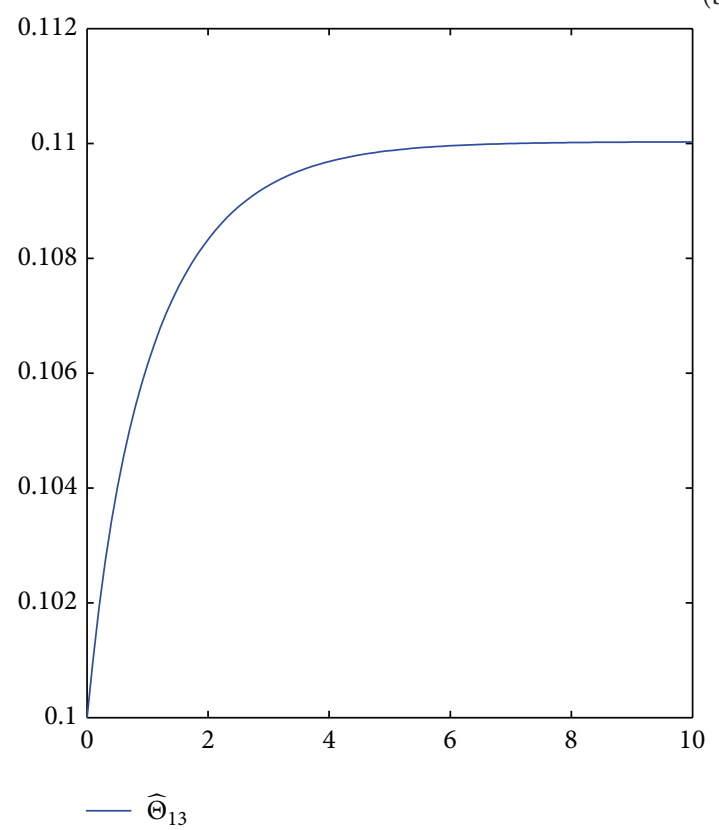

(c)

Figure 3: Parameters $\widehat{\Theta}_{11}, \widehat{\Theta}_{12}, \widehat{\Theta}_{13}$.

Define the invariable that $\eta_{1}(t)=z_{1}(t), \eta_{2}(t)=v_{2}(t)-$ $\alpha_{1}$. According to the iterative procedure in Section 3, we can design the virtual control function and controller $u_{1}(t)$ as

$$
\begin{aligned}
\alpha_{1}= & -\widehat{\Theta}^{T} \Upsilon_{1}=-\left[\widehat{\Theta}_{11}, \widehat{\Theta}_{12}, \widehat{\Theta}_{13}\right]\left[\Upsilon_{11}, \Upsilon_{12}, \Upsilon_{13}\right]^{T}, \\
u_{1}(t)= & -c_{2} \eta_{2}(t)+k_{2} v_{1}(t)+\frac{\partial \alpha_{1}}{\partial \widehat{\Theta}_{1}^{T}} \dot{\Theta}_{1}+\frac{\partial \alpha_{1}}{\partial \xi_{02}} \dot{\xi}_{02} \\
& +\frac{\partial \alpha_{1}}{\partial u_{0}(t)} \dot{u}_{0}(t)+\frac{\partial \alpha_{1}}{\partial z_{1}(t)}\left[\xi_{02}-\frac{\dot{u}_{0}(t)}{u_{0}(t)} z_{1}(t)\right]
\end{aligned}
$$

$$
\begin{aligned}
& -\frac{\ell_{2}}{4}\left(P_{12}^{2}+P_{22}^{2}\right)\left(\frac{\partial \alpha_{1}}{\partial z_{1}(t)}\right)^{2} \eta_{2}(t) \\
& -\frac{1}{2}\left(\frac{\partial \alpha_{1}}{\partial z_{1}(t)}\right)^{2} e^{2 x_{0}(t)} \eta_{1}^{2}(t) \eta_{2}(t)-\widehat{\Theta}_{2}^{T} \Upsilon_{2}
\end{aligned}
$$

where

$$
\begin{aligned}
\Upsilon_{11}= & c_{1} \eta_{1}(t)+\xi_{02}(t)+\frac{1}{2} \eta_{1}^{3}(t) e^{2 x_{0}(t)} \\
& +\left[\frac{\ell_{1}}{8}+\frac{\delta_{1}}{32}\|Q\|^{2}\right] \eta_{1}^{7}(t) u_{0}^{8}(t)
\end{aligned}
$$




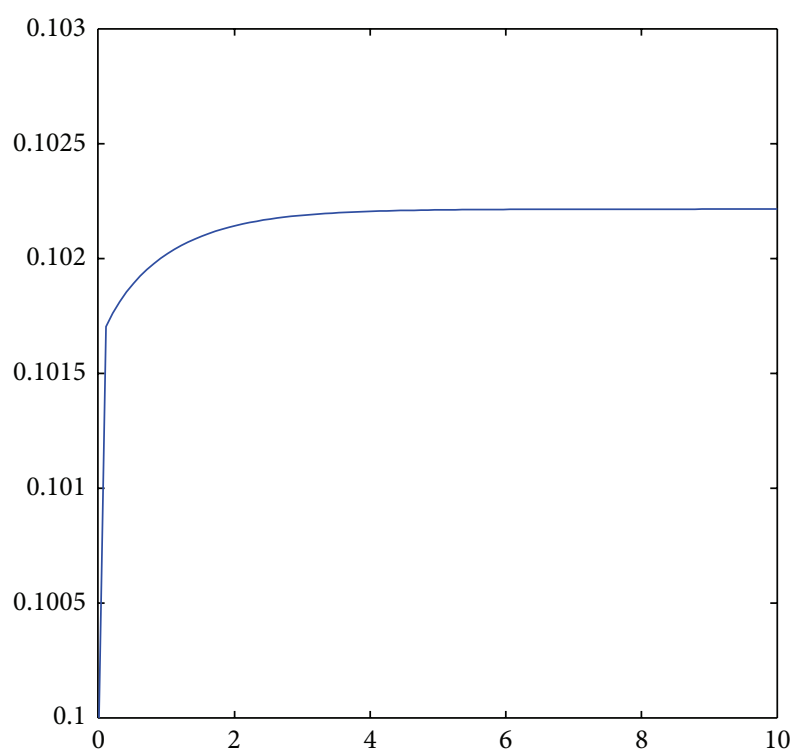

$-\widehat{\Theta}_{21}$

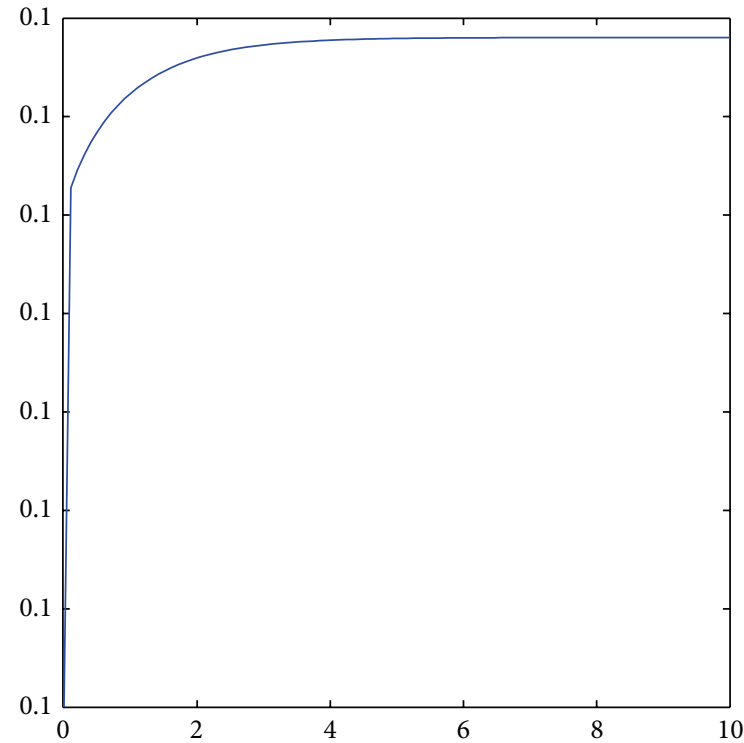

$-\widehat{\Theta}_{22}$

(b)

(a)

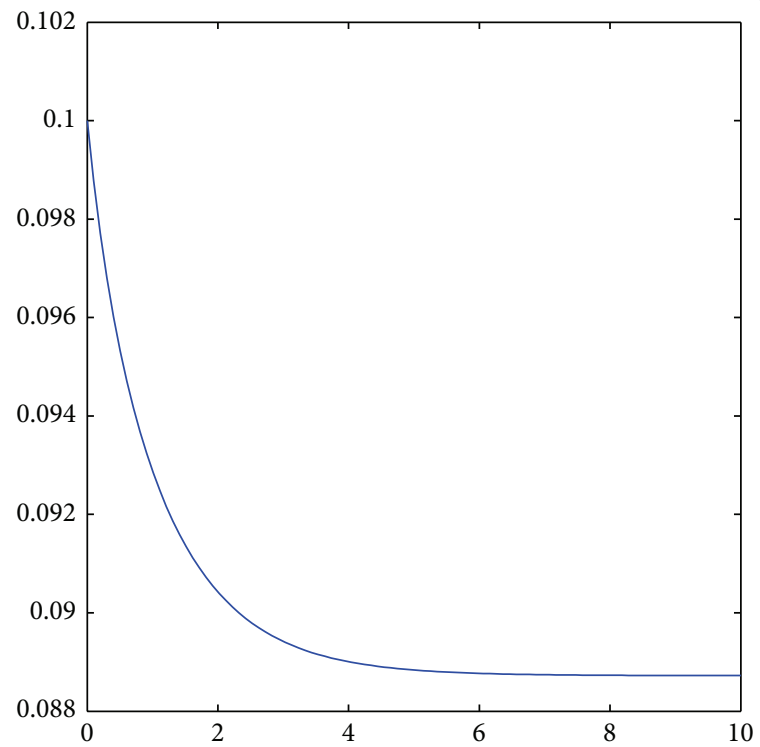

$-\widehat{\Theta}_{23}$

(c)

Figure 4: Parameters $\widehat{\Theta}_{21}, \widehat{\Theta}_{22}, \widehat{\Theta}_{23}$.

$+\left[2 \ell_{1}+\frac{\delta_{1}}{2}\|Q\|^{2}\right] \eta_{1}^{11}(t) e^{4 x_{0}(t)} u_{0}^{12}(t)+\frac{1}{4} \eta_{1}^{3}(t) u_{0}^{4}(t), \quad \widehat{\Theta}_{2}^{T}=\left[\widehat{\Theta}_{21}, \widehat{\Theta}_{22}, \widehat{\Theta}_{23}\right]$,

$$
\begin{array}{rlrl}
\Upsilon_{12}= & 2 \ell_{1} \eta_{1}^{3}(t) e^{4 x_{0}(t)}+2 \ell_{1} \eta_{1}^{3}(t) u_{0}^{4}(t) & \Upsilon_{2}=\left[\frac{1}{4}\left(\frac{\partial \alpha_{1}}{\partial z_{1}(t)}\right)^{2} e^{z_{1}^{2}(t) u_{0}^{2}(t)} \eta_{2}(t), \frac{1}{4} \eta_{2}(t),-\frac{\partial \alpha_{1}}{\partial z_{1}(t)} v_{2}(t)\right]^{T} . \\
& +\frac{\delta_{1}}{2}\|Q\|^{2} \eta_{1}^{3}(t) e^{4 x_{0}(t)}+\frac{\delta_{1}}{2}\|Q\|^{2} \eta_{1}^{3}(t) u_{0}^{4}(t),
\end{array}
$$

$\Upsilon_{13}=\left[\frac{1}{4}+4 \ell_{1}+\delta_{2}\|Q\|^{2}\right] \eta_{1}(t) e^{z_{1}^{2}(t) u_{0}^{2}(t)}$

The adaption laws of the parameter invariable in controller $u_{1}(t)$ are chosen as

$+\left[4 \ell_{1}+\delta_{2}\|Q\|^{2}\right] \eta_{1}(t) u_{0}^{2}(t)$,

$$
\dot{\widehat{\Theta}}_{1}=\operatorname{sign}\left(\bar{d}_{2}\right) \Upsilon_{1} \eta_{1}(t), \quad \dot{\widehat{\Theta}}_{2}=\Upsilon_{2} \eta_{2}(t) .
$$


For simulation use, we pick the unknown parameters $d_{1}=1.5, d_{2}=2.5, \theta_{1}=\theta_{2}=0.5$. In addition, we take the other controller design parameters as $c_{0}=1, c_{1}=$ $130, c_{2}=2, k_{1}=4, k_{2}=1, \ell_{1}=2, \ell_{2}=3, \delta_{1}=$ $\delta_{2}=4$. Moreover, The initial state condition is $[0.2,0,-0.1]^{T}$. Simulation results are shown in Figures 1, 2, 3, and 4. It is obvious that the states $x_{0}(t), x_{1}(t), x_{2}(t)$ and control input $u_{0}(t), u_{1}(t)$ converge to zero, and the parameters estimation invariable tend to constants.

\section{Conclusion}

The output-feedback adaptive stabilization was investigated for a class of nonholonomic systems with unknown virtual control coefficients, nonlinear uncertainties, and unknown time delays. In order to overcome the difficulties, we introduce suitable transformation and novel Lyapunov-Krasovskii functionals, and then a recursive technique is given to design the adaptive controller. To make the input-state scaling transformation effective, the switching control strategy is employed to achieve the asymptotic stabilization.

\section{Conflict of Interests}

The authors declare that there is no conflict of interests regarding the publishing of this paper.

\section{Acknowledgments}

This work is partially supported by National Natural Science Foundation (U1304620,61273091,61374079), Basic and Frontier Technologies Research Program of Henan Province (122300410279), and Doctoral Fund of Zhengzhou University of Light Industry (201BSJJ006).

\section{References}

[1] R. W. Brockett, "Asymptotic stability and feedback stabilization," in Differential Geometric Control Theory, R. W. Brockett, R. S. Millman, and H. J. Sussmann, Eds., vol. 27, pp. 181-191, Birkhäuser, Boston, Mass, USA, 1983.

[2] M. A. Krasnosel'skii and P. P. Zabreiko, Geometrical Methods of Nonlinear Analysis, Springer, Berlin, Germany, 1984.

[3] A. M. Bloch and N. H. McClamroch, "Control of mechanical systems with classical nonholonomic constraints," in Proceedings of the 28th IEEE Conference on Decision and Control, pp. 201-205, Tampa, Fla, USA, 1989.

[4] A. M. Bloch, M. Reyhanoglu, and N. H. McClamroch, "Control and stabilization of nonholonomic dynamic systems," IEEE Transactions on Automatic Control, vol. 37, no. 11, pp. 1746-1757, 1992.

[5] A. Astolfi, "Discontinuous control of nonholonomic systems," Systems \& Control Letters, vol. 27, no. 1, pp. 37-45, 1996.

[6] W. Lin, R. Pongvuthithum, and C. Qian, "Control of high-order nonholonomic systems in power chained form using discontinuous feedback," IEEE Transactions on Automatic control, vol. 47, no. 1, pp. 108-115, 2002.
[7] J. Luo and P. Tsiotras, "Control design for chained-form systems with bounded inputs," Systems \& Control Letters, vol. 39, no. 2, pp. 123-131, 2000.

[8] W. Lin, "Time-varying feedback control of nonaffine nonlinear systems without drift," Systems \& Control Letters, vol. 29, no. 2, pp. 101-110, 1996.

[9] R. T. M’Closkey and R. M. Murray, "Exponential stabilization of driftless nonlinear control systems using homogeneous feedback," IEEE Transactions on Automatic Control, vol. 42, no. 5, pp. 614-628, 1997.

[10] Y.-P. Tian and S. Li, "Exponential stabilization of nonholonomic dynamic systems by smooth time-varying control," Automatica, vol. 38, no. 8, pp. 1139-1146, 2002.

[11] Z. P. Jiang, "Iterative design of time-varying stabilizers for multi-input systems in chained form," Systems \& Control Letters, vol. 28, no. 5, pp. 255-262, 1996.

[12] Z.-P. Jiang, "Robust exponential regulation of nonholonomic systems with uncertainties," Automatica, vol. 36, no. 2, pp. 189209, 2000.

[13] O. J. Sørdalen and O. Egeland, "Exponential stabilization of nonholonomic chained systems," IEEE Transactions on Automatic Control, vol. 40, no. 1, pp. 35-49, 1995.

[14] Z. P. Jiang and H. Nijmeijer, "A recursive technique for tracking control of nonholonomic systems in chained form," IEEE Transactions on Automatic control, vol. 44, no. 2, pp. 265-279, 1999.

[15] S. S. Ge, Z. Wang, and T. H. Lee, "Adaptive stabilization of uncertain nonholonomic systems by state and output feedback," Automatica, vol. 39, no. 8, pp. 1451-1460, 2003.

[16] X. W. Mu, J. M. Yu, and G. F. Zhang, "Adaptive regulation of high-order nonholonomic systems," Applied Mathematics and Mechanics, vol. 27, no. 4, pp. 501-507, 2006.

[17] F. Gao, F. Yuan, and H. Yao, "Robust adaptive control for nonholonomic systems with nonlinear parameterization," Nonlinear Analysis: Real World Applications, vol. 11, no. 4, pp. 32423250, 2010.

[18] F. Gao, F. Yuan, J. Zhang, and Y. Wu, "Further result on finite-time stabilization of stochastic nonholonomic systems," Abstract and Applied Analysis, vol. 2013, Article ID 439482, 8 pages, 2013.

[19] Z. Xi, G. Feng, Z. P. Jiang, and D. Cheng, "A switching algorithm for global exponential stabilization of uncertain chained systems," IEEE Transactions on Automatic Control, vol. 48, no. 10, pp. 1793-1798, 2003.

[20] Z. P. Jiang, "Lyapunov design of global state and output feedback trackers for non-holonomic control systems," International Journal of Control, vol. 73, no. 9, pp. 744-761, 2000.

[21] Z. Xi, G. Feng, Z. P. Jiang, and D. Cheng, "Output feedback exponential stabilization of uncertain chained systems," Journal of the Franklin Institute, vol. 344, no. 1, pp. 36-57, 2007.

[22] Y. G. Liu and J. F. Zhang, "Output-feedback adaptive stabilization control design for non-holonomic systems with strong non-linear drifts," International Journal of Control, vol. 78, no. 7, pp. 474-490, 2005.

[23] G. Ju, Y. Wu, and W. Sun, "Adaptive output feedback asymptotic stabilization of nonholonomic systems with uncertainties," Nonlinear Analysis: Theory, Methods \& Applications, vol. 71, no. 11, pp. 5106-5117, 2009.

[24] X. Zheng and Y. Wu, "Adaptive output feedback stabilization for nonholonomic systems with strong nonlinear drifts," Nonlinear Analysis: Theory, Methods \& Applications, vol. 70, no. 2, pp. 904920, 2009. 
[25] Y. Wu and Y. Wu, "Robust stabilization of delayed nonholonomic systems with strong nonlinear drifts," Nonlinear Analysis: Real World Applications, vol. 11, no. 5, pp. 3620-3627, 2010.

[26] Y. Y. Wu and Y. Q. Wu, "Robust stabilization for nonholonomic systems with state delay and nonlinear drifts," Journal of Control Theory and Applications, vol. 9, no. 2, pp. 256-260, 2011.

[27] W. Lin and C. Qian, "Adaptive control of nonlinearly parameterized systems: the smooth feedback case," IEEE Transactions on Automatic Control, vol. 47, no. 8, pp. 1249-1266, 2002. 


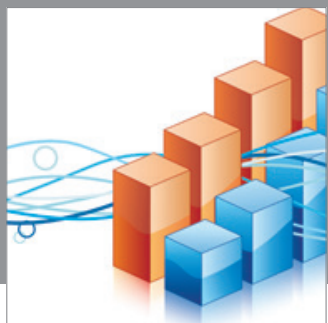

Advances in

Operations Research

mansans

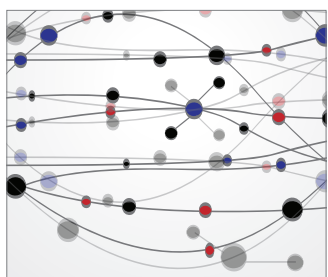

The Scientific World Journal
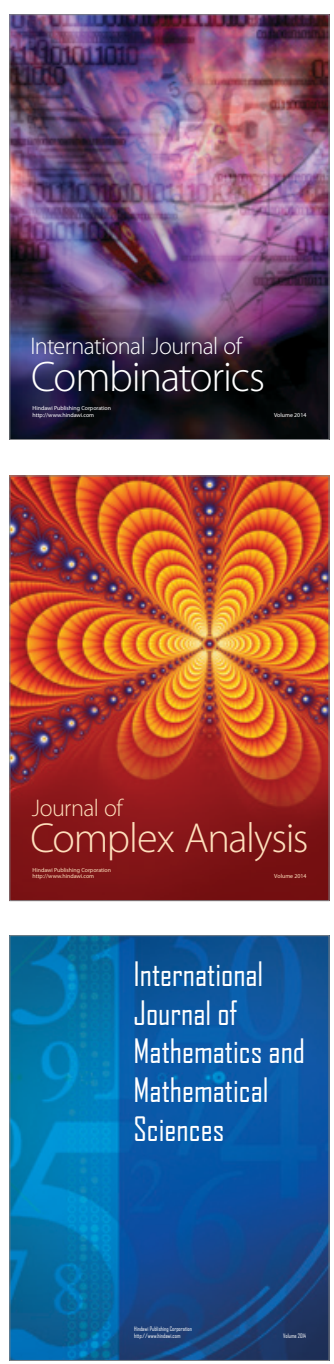
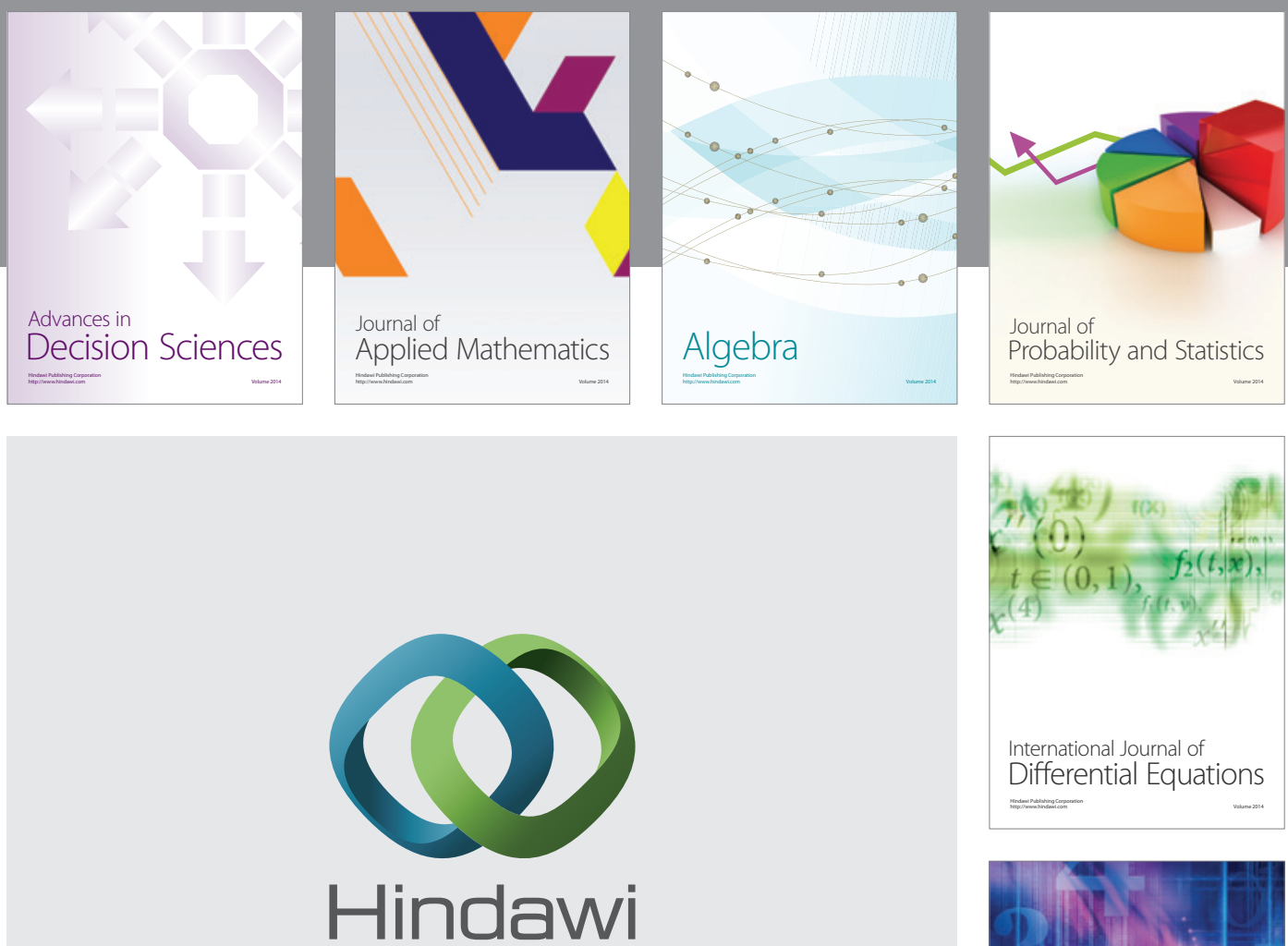

Submit your manuscripts at http://www.hindawi.com
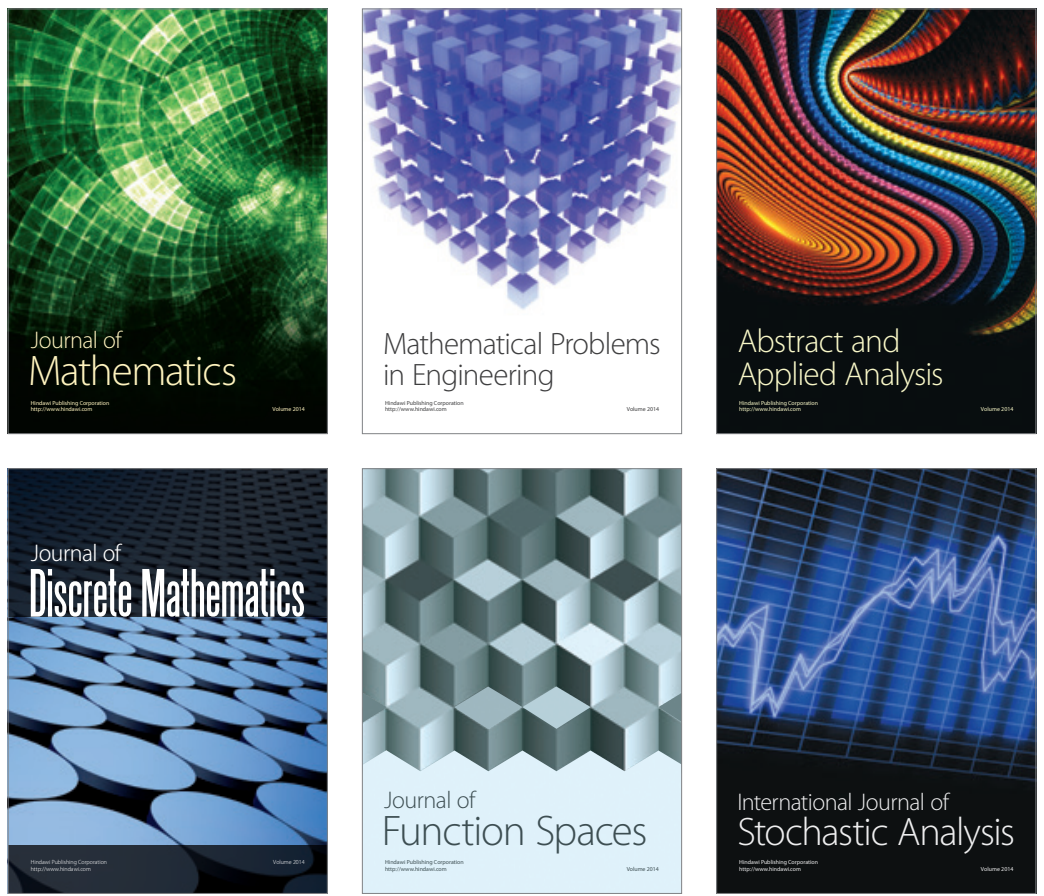

Journal of

Function Spaces

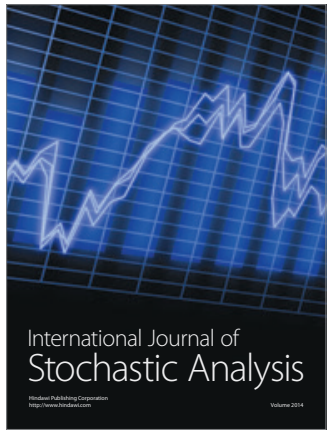

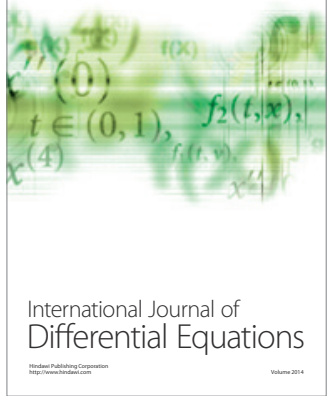
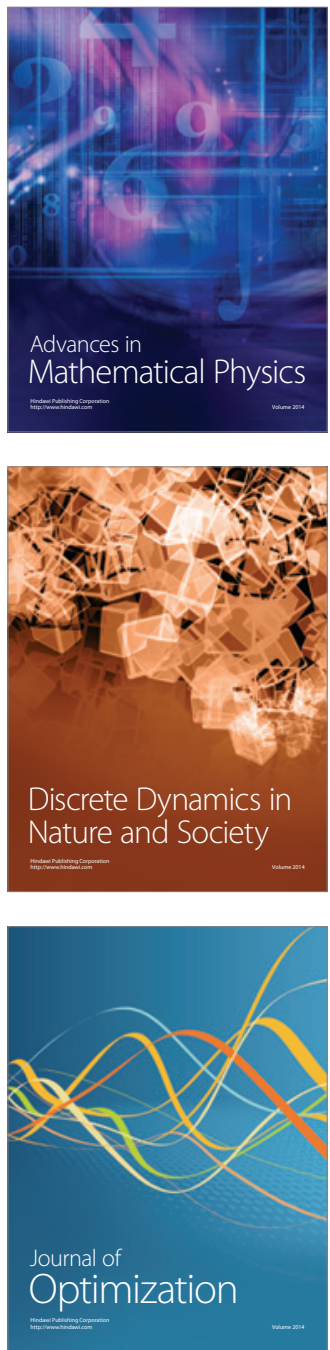